() Entomologica Fennica. 29 June 1994

\title{
Lepidoptera of the Kola Peninsula, northwestern Russia'
}

\author{
Michail V. Kozlov \& Jukka Jalava
}

Kozlov, M. V. \& Jalava, J. 1994: Lepidoptera of the Kola Peninsula, northwestern Russia. - Entomol. Fennica 5:65-85.

The annotated list of 585 species of Lepidoptera from the Kola Peninsula is the first comprehensive account from the area. It is based on results of a recent 14 years collecting as well as on the old materials collected by Finnish entomologists and on all the data published earlier. For each species, the localities are listed; position of all localities is indicated on the map; species numbers from localities and UTM squares are summarized. Eight species are new for Russia; six species collected along the White Sea coast were never recorded in Northern Fennoscandia. Eight species erroneously reported from the Kola Peninsula are excluded from the list.

Michail V. Kozlov, Laboratory of Ecological Zoology, University of Turku, FIN-20500 Turku, Finland

Jukka Jalava, Finnish Museum of Naturl History, P.O.Box 17, FIN-00014

University of Helsinki, Finland

Received 16 March 1993, accepted 2 June 1993

\section{Introduction}

The territory of the Kola Peninsula belongs geographically to Northern Fennoscandia, the Lepidopteran fauna of which is quite well studied. Comprehensive reviews have been published for Finnish Lapland (Krogerus 1972, Koponen et al. 1982, Linnaluoto \& Koponen 1980) and for the Finnmark area in Norway (Opheim 1975, 1977, 1978, Aagaard 1979). Compared with these areas, Kola Peninsula has practically been a "white spot", except the old Finnish territory (Petsamo area: Valle 1933, northern parts; Krogerus 1943, southern parts).

Most of the materials collected by Finnish entomologists in central, southern and eastern Kola in the last and at the beginning of this century (see

\footnotetext{
${ }^{1}$ Report no. 1 from the research project on the Entomological Bioindicators on Kola Peninsula
}

Silfverberg 1988 for history) are deposited in the Zoological Museum in Helsinki (MZH), but hardly any results have been published earlier. However, Tengström $(1869,1873)$ published some records. Two short lists are known from the Murmansk area (Djakonov 1911, Miller 1915). Some species were reported from Rybatshij peninsula (Kotzsch 1933). Ecology of insect communities in the Chibiny mountains was studied by Fridolin $(1935,1936)$, but only a few species of Lepidoptera (especially butterflies) were mentioned. In his fauna of the northem parts of Russian Carelia, Peltonen (1947) published a few records from Kouta (our points 62 and 66). Recently fragmentary data have been published in taxonomic (e.g. Nielsen \& Johansson 1980), faunistic (Kozlov 1981a, 1983a, b, c, 1984a, 1987, Sinev 1988) and applied (Znamenskaja 1962, Novitskaja 1962, Vershinina 1981, Kozlov 1981b, 1984b) papers. Some records are mentioned in faunistic reviews of different taxonomic groups (e.g., Nordström et al. 1955, 1961, 1969). 


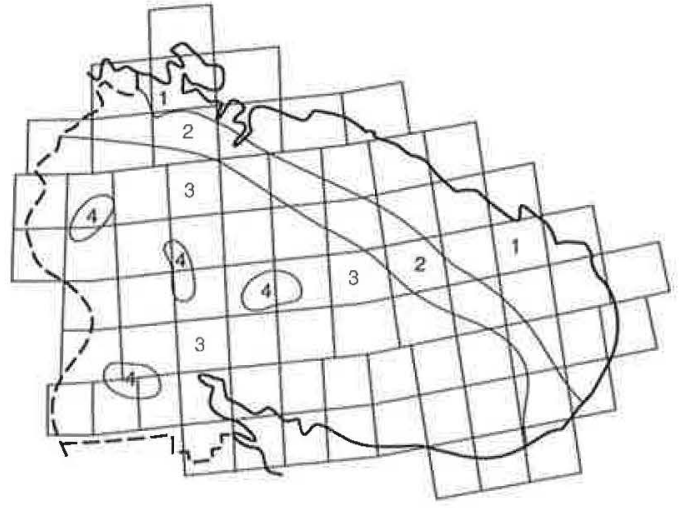

Fig. 1. Zonal types of vegetation on Kola Peninsula (after Gribova et al. 1980): 1 - southern tundra, 2 birch woodlands, 3 - northern taiga, 4 - mountain tundra.

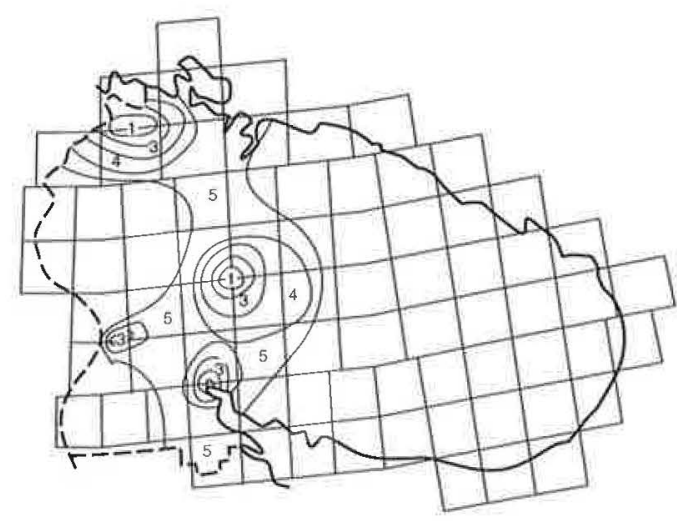

Fig. 2. Pollution zones on Kola Peninsula (after Kryuchkov 1991): 1 - industrial barren, 2 - secondary birch forests, 3 - destroyed coniferous forests, 4 damaged forests, 5 - initial step of deterioration.

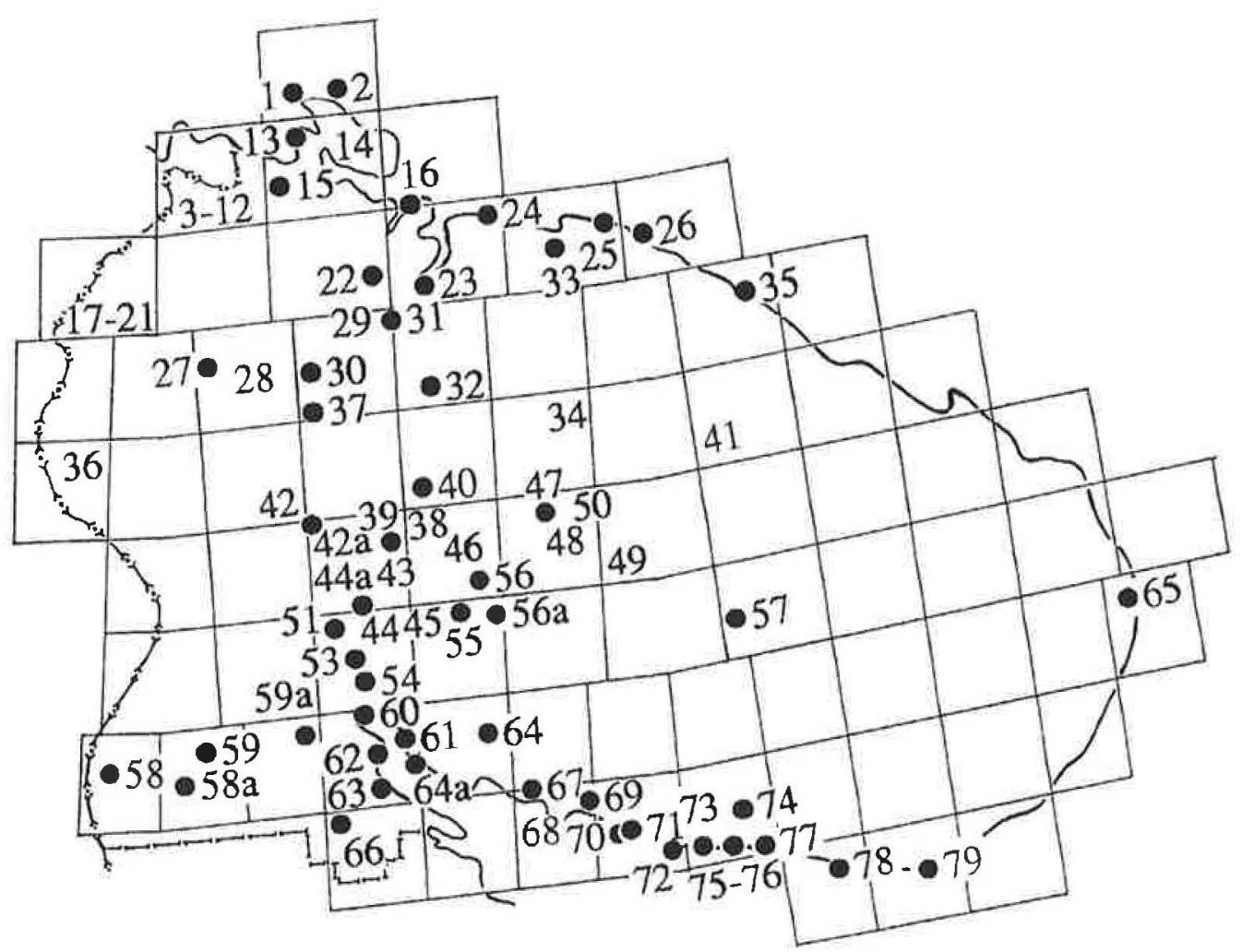

Fig. 3. Position of the localities on the Kola Peninsula (see Table 1 for explanation of numbers): black circles localized sites; other figures - large sample areas. 
Table 1. The collecting localities (numbered for reference to map in Fig. 3), their position within UTM squares and biogeographical provinces, and number of species found.

\begin{tabular}{|c|c|c|c|c|c|c|c|c|c|}
\hline No & Locality & UTM & Province & Species & No & Locality & UTM $\mathrm{Pr}$ & Province & Species \\
\hline 1 & Kervanto & VC3 & Lps & 39 & 43 & St.Petersburg road S of $39^{1}$ & VA4 & Lim & 205 \\
\hline 2 & Vaitolahti & VC3 & Lps & 52 & 44 & Lapland reserve $^{2}$ & VA4 & Lim & 174 \\
\hline 3 & Trifona & VC2 & Lps & 45 & $44 a$ & Tshuna-tundra mt. & VA4 & Lim & 73 \\
\hline 4 & Liinahamari & VC2 & Lps & 56 & 45 & Jokostrov (Ekostroff) & WV1 & Lim & 35 \\
\hline 5 & Kuvernöörinkoski & VC2 & Lps & 65 & 46 & Chibiny mt. & WA2 & $\operatorname{Lim}$ & 163 \\
\hline 6 & Parkkino & VC2 & Lps & 71 & 47 & Revda & WA4 & Lmur & 5 \\
\hline 7 & Näsykkä & VC2 & Lps & 1 & 48 & Lovozero mt.(Lujaururt) & WA4 & Lmur & $r \quad 135$ \\
\hline 8 & Haukilampi & VC2 & Lps & 123 & 49 & Marjok & XA2 & Lmur & 9 \\
\hline 9 & Salmijärvi & VC2 & Lps & 148 & 50 & Niemlomjok & WA4 & Lmur & 7 \\
\hline 10 & Yläluostari & VC2 & Lps & 184 & 51 & Upoloksha & VV3 & Lim & 11 \\
\hline 11 & Zapolyarny & VC2 & Lps & 3 & 52 & surr. of Imandra lake & N3-WA2 & $2 \mathrm{Lim}$ & 34 \\
\hline 12 & Petchenga (Petsamo) & VC2 & Lps & 205 & 53 & Zasheek (Saseika) & VV3 & Lim & 35 \\
\hline 13 & Heinäsaaret & VC3 & Lps & 23 & 54 & Poljarnye Zori & VV3 & Lim & 3 \\
\hline 14 & Rybatshij & VC4 & Lps & 44 & 55 & Apatity & WV1 & Lim & 165 \\
\hline 15 & Pummanki & VC4 & Lps & 103 & 56 & Kirovsk & WA2 & Lim & 128 \\
\hline 16 & Eretik & WB1 & Lt & 2 & $56 a$ & 15 km E Kirovsk & WV1 & $\operatorname{Lim}$ & 45 \\
\hline 17 & Pasvik (=Patsjoki) & NS3 & Lps & 31 & 57 & Krasnoschelje & DQ1 & Lv & 2 \\
\hline 18 & Nautsi & NS3 & Lps & 100 & 58 & Kuolajärvi & PQ2 & Ks & 6 \\
\hline 19 & Kolttaköngäs & NS3 & Lps & 27 & $58 a$ & Vuorijärvi & UV & $\mathrm{Ks}$ & 81 \\
\hline 20 & Höyhenjärvi & NS3 & Lps & 1 & 59 & Alakurtti & UV & Ks & 8 \\
\hline 21 & Pitkäjärvi & NS3 & Lps & 68 & $59 a$ & 35 km W Kandalaksha & VV2 & Lim & 27 \\
\hline 22 & Kilp-javr & VB3 & Lt & 12 & 60 & Kandalaksha & VV4 & Lim & 134 \\
\hline 23 & Murmansk & WB1 & Lt & 83 & 61 & Luvenga & VV4 & Lim & 108 \\
\hline 24 & Kildin & WB1 & $\mathrm{Lt}$ & 1 & 62 & Knjazhaja Guba & VV4 & $\mathrm{Kk}$ & 19 \\
\hline 25 & Gavrilovo & WB3 & Lmur & 3 & 63 & Zelenoborskij & VV4 & $\mathrm{Kk}$ & 45 \\
\hline 26 & Dalnije Zelentsy & DS1 & Lmur & 3 & 64 & Konosero & WV2 & Lim & 17 \\
\hline 27 & Köngäs & NR3 & Lps & 61 & $64 a$ & Kolvitsa & V44 & Lim & 75 \\
\hline 28 & Lutto & NR3 & Lps & 155 & 65 & Ponoj & FQ2 & Lp & 69 \\
\hline 29 & Tuloma & VB4 & $\mathrm{Lt}$ & 18 & 66 & Kouta & VU3 & Kk & 19 \\
\hline 30 & Verhnetulomskij & VB2 & $\mathrm{Lt}$ & 30 & 67 & Umba & WU3 & Lim & 89 \\
\hline 31 & Kola & VB2 & $\mathrm{Lt}$ & 67 & 68 & Turij, 20 km E Umba & WU3 & Lim & 30 \\
\hline 32 & Loparskaja & WB2 & Lim & 7 & 69 & Kusräka & WU3 & LV & 22 \\
\hline 33 & Tumannyi & WB3 & Lmur & 2 & 70 & $45 \mathrm{~km} \mathrm{E} \mathrm{Umba}{ }^{3}$ & XU1 & Lv & 44 \\
\hline 34 & Voronja r. & WA3 & Lmur & 42 & 71 & Olenitsa & $\mathrm{XU1}$ & Lv & 26 \\
\hline 35 & Kharlovka & DS2 & Lmur & 3 & 72 & 74 km E Umba ${ }^{3}$ & XU1 & Lv & 11 \\
\hline 36 & Saariselkä & NR3 & Lps & 42 & 73 & Kashkarantsy & CP1 & Lv & 105 \\
\hline 37 & Nuortjärvi & VB2 & $\mathrm{Lt}$ & 36 & 74 & Varzuga & CP1 & Lv & 2 \\
\hline 38 & St.Petersburg road $\mathbf{N}$ of $39^{1}$ & WA2 & $\operatorname{Lim}$ & 96 & 75 & $128 \mathrm{~km} \mathrm{E} \mathrm{Umba}{ }^{3}$ & CP1 & Lv & 33 \\
\hline 39 & Monchegorsk & VA4 & Lim & 69 & 76 & $132 \mathrm{~km} \mathrm{E} \mathrm{Umba}{ }^{3}$ & CP1 & Lv & 24 \\
\hline 40 & Olenegorsk & WA1 & $\operatorname{Lim}$ & 17 & 77 & Kuzomen & CP1 & Lv & 71 \\
\hline 41 & Tshilozero & DR1 & Lmur & 1 & 78 & Tshavanga & DP2 & Lv & 4 \\
\hline 42 & Njavka & VA4 & $\mathrm{Lim}$ & 1 & 79 & Tetrino & DP4 & Lv & 3 \\
\hline $42 a$ & Monche-tundra mt. & VA4 & Lim & 50 & & & & & \\
\hline
\end{tabular}

${ }^{1}$ Localities 38 and 43 represent lines of sampling sites along two pollution gradients directed $\mathrm{N}$ and $\mathrm{S}$ of Monchegorsk, along the road St.Petersburg-Murmansk. In the list of species, distance (in kms) from the smelter is added to the locality number after a hyphen. $-{ }^{2}$ Central country (Tshuna = Zuna), $40 \mathrm{~km} \mathrm{SSW}$ Monchegorsk. - ${ }^{3}$ Material collected on the White Sea coast, outside of any settlements.

Our goal was to collect all the information available from different sources and to prepare the first list of Lepidoptera of the Kola Peninsula necessary for comparative analysis of Fennoscandian fauna of moths and butterflies as a whole. The second aim of the paper is to create a base for the environmental monitoring programs in polluted areas around different smelters on the Peninsula. 


\section{Materials and methods}

\subsection{The study area}

The Kola Peninsula is situated in the northwesternmost corner of Russia (from 66 to $70^{\circ} \mathrm{N}$ and from 28 to $41^{\circ} \mathrm{E}$ ). Following administrative borders, we use the name "Kola Peninsula" as an equivalent of Murmansk region ("Murmanskaya oblast") in Russia, although in a strict geographical sense neither the western nor the southern areas belong to the Peninsula. Thus, the study area was $144900 \mathrm{~km}^{2}$. In the west it is bordered by Finland and Norway, in the north by the Arctic Ocean and in the east and south by the White Sea.

An east-west directed watershed through the middle parts of the Peninsula divides the area into two different regions. Southwards of the watershed, coniferous forests (Picea and Pinus) cover most of the territory. Northern and eastern parts are mostly covered by mountain birch (Betula tortuosa) or northern tundra, the eastern parts being mostly tundra (Fig. 1). High fells are situated in the central and western parts. The highest peaks are in the middle of the Peninsula: Chibiny mountains (1190 $\mathrm{m})$, Lovozerskie tundra $(1120 \mathrm{~m})$ and Tshuna-tundra (1114 $\mathrm{m}$ a.s.l.). These mountain massives are formed of alkaline phosphate minerals and have a very rich alpine flora (Ramenskaja 1983). The altitudinal timber line (formed by B. tortuosa) is at about 350$400 \mathrm{~m}$ a.s.l. The southern coastal areas have a marine vegetation (e.g. Aster tripolium is very abundant) and a marine climate as the White Sea is mostly open during the winter time.

The main sources of aerial pollution are copper-nickel smelters situated in Nikel, Zapolyarny and Monchegorsk; less powerful sources of emission are situated in Murmansk, Olenegorsk, Apatity, Kirovsk, Kovdor and Kandalaksha. As a result, practically all territories situated along the road St. Petersburg-Murmansk (where most of the materials has been collected) belong to polluted zones (Fig. 2). For more details, see Kryuchkov (1993).

\subsection{Collections}

The main material was collected by M. Kozlov in 1980-1993 and by J. Jalava, A. Lvovsky and L. Svetsova in 1991 and 1992. All the habitats - different types of forests (pine, spruce, mixed and birch), marshes, meadows along the White Sea shore and along the big rivers, subalpine birch forests, mountain tundra, ruderal areas around towns etc. - were investigated. The moths were collected mostly by netting in the day time and evenings; some species were captured during special excursions at evenings and nights (Noctuidae in spruce forests) or early in the morning at dawn (Microlepidoptera). Some records of Nepticulidae are based on mines only. Rearing from larvae was not widely used. About 10 species of leafrollers were collected by pheromone traps "Atracon A" with different types of lure; sugar bait-traps were used for noctuid moths. Collections are mostly deposited in MZH; a part of material collected by $\mathrm{M}$. Kozlov (mostly Tortricidae, Oecophoridae and Geometridae) is kept in the Zoological Institute, St. Petersburg, Russia (ZIN).

All the materials collected by Finnish entomologists (deposited in $\mathrm{MZH}$ ) were investigated, and the records are included in our list. We have also controlled some determinations in the collections of K. J. Valle (kept in Turku University, Finland) and V. Ju. Fridolin (kept in ZIN). Totally about 15000 specimens were taken into consideration.

Numerous records of "Macrolepidoptera" not supported by original material were extracted from the unpublished data of the late Prof. O. Sotavalta who has collected distribution records from Eastern Fennoscandia for decades. These data are now kept in the Finnish Lepidopterological Society (Helsinki) and will be partly published in the nearest years.

The species are listed below in taxonomic order according to the Finnish check-list (Varis et al. 1987). For each species, localities are given in a numerical sequence. For the species known from one to two localities only, or having a special importance for the discussion, number of specimens and date of collectiing are given. If the date of collecting is established by the timeschedules of the trips of Finnish entomologists (Silfverberg 1988), it is shown within square brackets. Collector name is given only for the specimens having incomplete information on the label (mostly from old Finnish collections).

Within the list of localities, references are used if we have not seen any specimens from the locality, except the most common species where no references are given. 


\subsection{Place names}

We have used locality names from recent Russian maps of Murmansk district. Certain old names have been replaced by new ones, using both the publication (Silfverberg 1988) and direct consultations with Dr. H. Silfverberg. Position in the UTM system was marked following Jalas \& Suominen (1972). All the localities are listed in Table 1; their position within Kola Peninsula is shown on Fig. 3. Localities are numbered from NW to SE on the map, following the rows of UTM squares.

Since the material collected in 1993 was added in proof, six localities received additional numbers with a letter.

\section{List of species}

\section{Micropterigidae}

Micropterix aureatella (Scop.). 3, 9, 10, 12, 23, 31, 34, $43-29,43-15,44,46,48,53,55,56,58 a, 59 a, 60,61$, 64a, 67, 73 .

Micropterix mansuetella Zell. 43-29: 1 ex 8.7.1991.

Micropterix calthella (L.). 55: $4 \operatorname{exx}$ 30.6.1991. The northernmost record.

\section{Eriocraniidae}

Eriocrania sparrmannella (Bosc). 18, 21, 31, 38-14, 4329, 43-19, 43-14, 44, 46, 55, 56, 60, 61, 73. Mostly reported on the base of mines which are easy to separate from those of other Eriocrania species.

Eriocrania salopiella (Stt.). 10: 2 exx 27.5.1979, 1 ex 2.6 .1980

Eriocrania sangii (Wood). 4, 38-14, 43-29, 43-17, 43-3, $44,46,55,56,60,61,73$. Mostly reported on the base of mines with grey caterpillars.

Eriocrania semipurpurella (Stph.). 10, 12, 23, 29, 38-8, $39,40,43-29,43-17,44,46,48,55,56,60,61,63$, $67,73,77$. Mostly reported on the base of mines.

\section{Hepialidae}

Hepialus hecta (L.). 10, 45, 60 .

Hepialus fusconebulosus (DeGeer). 5, 9, 12, 15, 17, 46, $56,61,71,73$.

Hepialus ganna (Hb.). 9 (Valle 1933), 46 (Fridolin 1936), 50 (Valle 1933), 65: 1 ex [1899] (Montell leg.), 74 (Nordström et al. 1961).

\section{Nepticulidae}

Stigmella lapponica (Wck.), 10, 44 (mines on Betula tortuosa), 44a, 55.
Stigmella betulicola (Stt.). 44 (mines on Betula nana).

Stigmella nylandriella (Tengstr.). 44, 56, 60, 61 (mines on Sorbus aucuparia).

Stigmella salicis (Stt.). 39: 13 exx 9.7.1993, 61: 1 ex 25.7.1991.

Stigmella zelleriella (Snell.). 55: 1 ex 17.7.1991 (peat bog).

Stigmella sorbi (Stt.). 12, 23, 38-10, 39, 43-29, 43-17, $43-7,44,46,48,55,56,60,61,67,73,77$.

Stigmella aeneofasciella (H.-S.). 46 (Raswumchorr, 600 m): 2 exx 1.8.1991.

Stigmella dryadella (Hofm.), 46 (Kukis-wum-chorr, 900 m, mines), 48 (Kuftuaj, 700 m): 2 exx 15.7.1991 (flying in the afternoon sunshine).

Ectoedemia weaveri (Stt.). 28: 2 exx [1939] (Platonoff leg.), 44a: 1 ex 15.7.1993.

Ectoedemia occultella (L.). 43-7: 1 ex 8.7.1992, 61: 1 ex 25.7.1991.

\section{Adelidae}

Nematopogon pilellus (Den. et Schiff.). 9: 1 ex 3.7.1930, 18: 2 exx 4-12.7.1929, 21: 1 ex 30.6.1928 (metaxellus Hb.: Valle 1933), 60: 1 ex [1870] (Sahlberg leg.).

Nematopogon schwarziellus (Z.). 61: 1 ex 28.7.1991, 73: 9 $\operatorname{exx} 25.6 .1985$.

Nematopogon magnus (Z.). 27 (Krogerus 1943).

Nematopogon swammerdamellus (L.). 44: 1 ex 19.7.1981.

Nematopogon robertellus (Cl.). 12 (Valle 1933), 44: 6 exx 9-17.7.1981.

Nemophora amurensis (Alph.). 61, 64, 67, 71, 73 (Kyrki 1981a).

Nemophora esmarkella (Wck.). 5, 9, 10, 12, 34, 41 (Kyrki 1981a), 59a.

Adela cuprella (Den. et Schiff.). 31: 1 ex [8-29.6.1887] (Palmén leg.), 38-15: 1 ex 22.6.1993, 58: 1 ex (C. Nyberg leg.).

Cauchas rufimitrella (Scop.). 1: 1 ex [1928] (Hellen leg.). The northernmost record. This southern species is distributed in Finland only up to the province Kb (Kyrki 1978). There exists a small possibility that the specimen is mislabelled.

Cauchas breviantennella Nielsen et Johansson. 65: 1 ex [1899] (Montell leg., paratype, in MZH).

Phylloporia bistrigella (Hw.). 43-7: 1 ex 11.7.1992.

Incurvaria praelatella (Den. et Schiff.). 10, 12, 19, 43-29, $45,46,55$.

Incurvaria oehlmanniella (Hb.). 8, 9, 10, 12, 28, 44, 44a, $58 \mathrm{a}, 61$.

Incurvaria vetulella (Zett.). 8, 9, 10, 12, 14, 18, 44, 44a, $48,61,67,73$. For the differences between this species and $I$. circulella (Zett.) see Laasonen et al. (1981).

Incurvaria circulella (Zett.). 10 (Valle 1933), 28, 44a, 48, 61.

Incurvaria pectinea Hw. 37: 1 ex [1899] (Poppius leg.), 55: 1 ex 12.6.1993.

Alloclemensia mesospilella (H.-S.). 27 (Krogerus 1943), 44: 1 ex 1.7.1981. 
Lampronia luzella (Hb.). 28 (Krogerus 1943), 43-29, 44, 55, 58a, 62, 67.

Lampronia rupella (Den. et Schiff.). 8, 10, 12, 17, 43-5, $44,46,48,55,56,58 \mathrm{a}, 65,67$.

\section{Psychidae}

Lypusa maurella (Den. et Schiff.). 9, 10 (Valle 1933), 18, 60.

Dahlica lazuri (Cl.). 10, 12, 28, 31, 37, 68, 73.

Taleporia borealis (Wck.). 12 (Valle 1933), 34 (Valle 1933).

Taleporia tubulosa (Retz.). 64a: 2 exx 21.7.1993, 58a: 1 ex 4.7.1993, 70: 1 ex 15.7.1992.

Psyche sp. 43-29. Larval case which can belong to $P$. casta (Pall.) or $P$. norvegica (Schöyen).

Phalacropterix graslinella (Boisd.). 43-16 (larval case).

Sterrhopterix standfussi (Wck.). 5 (hirsutella: Valle 1933), 9 (larval case), 10, 31, 39, 43-29 (larval cases), 60 (hirsutella: Nordström et al. 1961).

\section{Tineidae}

Montescardia tessulatella (Lienig et Z.). 27 (Krogerus 1943), 28, 43-29, 43-23, 58a, 60, 64a, 70.

Myrmecozela ochraceella (Tengstr.). 27 (Krogerus 1943), 38-13: 1 ex 3.7.1991.

Haplotinea insectella (F.). 44: 1 ex 1.8.1980 (synantropic), 46: 1 ex 15.7.1936 (in ZIN).

Nemapogon cloacellus (Hw.). 6, 8, 10, 12, 23, 28, 43-23, 43-20, 44, 46, 48, 55, 61.

Nemapogon clematellus (F.). 44: 1 ex 19.7.1981.

Nemapogon picarellus (Cl.). 8, 27 (Krogerus 1943), 28.

Archinemapogon yildizae Koçak. 12 (Valle 1933), 27 (Krogerus 1943), 28, 43-14, 43-3, 64a.

Triaxomera fulvimitrella (Sodoffsky). 8, 18 (Valle 1933), 43-41, 43-29, 43-14, 45, 56.

Monopis laevigella (Den. et Schiff.). 8, 9, 10, 12, 28, 4329, 44, 46, 48, 69.

Monopis weaverella (Scott). 4, 21, 28, 43-23, 44, 44a, 61, $64 \mathrm{a}, 76$.

Monopis spilotella (Tengstr.). 8, 9, 10, 15, 18, 27, 28, 43$3,44 a, 65$.

Monopis monachella (Hb.). 65: 4 exx [1899] (Montell leg.).

Trichophaga scandinaviella Zag. 1; 1 ex 22.7.1929, 15: 1 ex 7.8.1929. The records of Trichophaga from Petsamo area were formerly (Valle 1933) considered to represent the species $T$. tapetzella (L.). Their identity was cleared out by Jalava \& Kyrki (1980).

Tineola bisselliella (Hummel). 10: 1 ex 17.5.1980, 39: 2 exx 24.7.1981, 55: 3 exx 31.7.1993 (synantropic).

\section{Gracillariidae}

Caloptilia betulicola (M.Hering). 58a: 2 exx 3-6.7.1993, 67: 2 exx 20.6.1985, 73: 1 ex 20.6.1985.

Caloptilia stigmatella (F.). 28 (Krogerus 1943).

Parornix loganella (Stt.). 6, 9, 12, 18, 21, 44, 55.
Parornix anglicella (Stt.). 55: 1 ex 5.7.1991,

Parornix betulae (Stt.). 10, 12, 21, 31, 32, 43-29, 43-15, $43-3,44$.

Parornix scoticella (Stt.). 55, 56, 58a.

Parornix polygrammella (Wck.). 2, 9, 10, 15, 28, 43-29, $44,46,48,55,60,73$.

Callisto coffeella (Zett.). 12, 15, 31, 36, 42a, 44, 46 (up to $500 \mathrm{~m}), 55,60,73$.

Phyllonorycter sorbi (Frey). 48, 56, 61.

Phyllonorycter junoniellus (Z.). 55: 1 ex 18.7.1991, 61: 1 ex 30.6.1989.

Phyllonorycter salicicolellus (Sircom). 55: 2 exx 17.7.1991, 56: 3 exx 12-13.6.1992, 56a: 1 ex 24.6.1993.

Phyllonorycter rolandi (Svensson). 10: 1 ex 15.6.1980, 56: 1 ex 12.6.1992.

Phyllonorycter hilarellus (Zett.). 9, 28, 36, 38-3, 39, 4329, 43-20, 43-9, 44, 48, 55, 56, 58a, 61.

Phyllonorycter cavellus (Zell.). 55 (new airport): 1 ex 13.6.1993.

Phyllonorycter strigulatellus (Lienig et Z.). 9 (Valle 1933), 10: 1 ex 30.6.1979 \& 10 exx 5-15.6.1980, 12 (Valle 1933), 55: 5 exx 13.6.1992.

Phyllonorycter anderidae (Fletcher). 10, 38-15, 43-20, 55, 58 a.

Phyllonorycter ulmifoliellus (Hb.). 4, 10, 12, 31, 39, 43$29,44,46,55,56,73$.

Phyllocnistis labyrinthella (Bjerk.). 55 (airport): mines on natively growing trees of Populus tremula, 8.8.1993.

\section{Roesslerstammiidae}

Roeslerstammia erxlebella (F.). 55: 1 ex 2.7.1991.

\section{Bucculatricidae}

Bucculatrix cristatella Z. 56: 13 exx 13-16.7.1993, 60: 1 ex [1870] (Sahlberg leg.).

Bucculatrix maritima Stt. 61: 18 exx 23-27.7.1991 (very abundant). A sea shore species monophagous on Aster tripolium. Distributed on the coasts of Finland northwards to the province PPe (Kyrki 1978). New for Russia; for futher information, see Seksayeva (in press).

\section{Douglasiidae}

Tinagma dryadis Stgr. 44a: 1 ex 12.8.1993, 48 (S slope, $700 \mathrm{~m}$ ): 6 exx 15.7.1991 (common).

\section{Yponomeutidae}

Yponomeuta evonymellus (L.). 39, 43-29, 43-20, 43-15, 43-1, 46, (Fridolin 1936), 64a.

Swammerdamia caesiella (Hb.). 8, 9, 12, 18, 29, 38-1, 38$11,38-15,43-14,43-8,44,48,53,56,63$.

Swammerdamia passerella (Zett.). 13, 18 (Valle 1933), $28,38-13,43-14,56$.

Paraswammerdamia lapponica (W.Petersen). 8: 1 ex 8.7.1931, 55 (peat bog, common at dawn in 1991). 
Paraswammerdamia conspersella (Tengstr.). 2, 5, 6, 8, 9, $10,12,15,18,28,31,34,38-11,42 \mathrm{a}, 43-29,43-23$, $43-14,43-8,43-3,44,44 a, 46,55,62,65,73$.

Cedestis gysseleniella Z. 62: 1 ex 11.8.1987.

Cedestis subfasciella (Stph.). 55: 1 ex 18.7.1991, 62: 1 ex 11.8.1987.

Ocnerostoma friesei Svensson. 38-15: 1 ex 22.6.1993, 44: 1 ex 22.6.1981.

Atemelia torquatella (Lienig et Z.). 56: 1 ex 12.6.1992.

\section{Argyresthiidae}

Argyresthia glabratella Z. 3, 43-29, 43-23, 43-13, 43-3, $44 \mathrm{a}, 45,48,55,58 \mathrm{a}, 70,76$.

Argyresthia bergiella Ratz. 43-26: 1 ex 4.7.1987, 44: 2 exx 19-27.7.1981.

Argyresthia abdominalis Z. 44: 1 ex 19.7.1981.

Argyresthia pygmaeella (Den. et Schiff.). 3, 5, 10, 12, 23, $38-15,38-11,39,43-41,43-9,43-5,43-1,44,56$, $61,64 a$.

Argyresthia sorbiella (Tr.). 44, 48, 55, 56, 60, 73.

Argyresthia conjugella Z. 43-29: 1 ex 27.7.1993, 56: 1 ex 3.7.1989 \& $3 \operatorname{exx} 20.7 .1991,61$ : 1 ex 25.7.1991.

\section{Plutellidae}

Plutella xylostella (L.). 10, 12, 23, 28, 32, 38-14, 39, 40, 42a, 43-29, 43-20, 43-12, 43-7, 43-1, 44, 44a, 46, $48,53,55,56,58,58 \mathrm{a}, 60,61,63,64 \mathrm{a}, 67,68,73,75$, 77.

Rhigognostis senilella (Zett.). 10: 6 exx 12.4-27.5.1980, $46(500 \mathrm{~m}): 2$ exx 18.7.1985, 48 (700 m): 1 ex 4.7.1986 \& 2 exx 15.7.1991.

Rhigognostis kuusamoensis Kyrki, 48 (Seitjaur): 1 ex 6.7.1986, 55: 2 exx 24.5-2.6.1993. So far recorded in Northern Fennoscandia only. For identity and distribution, see Kyrki (1988).

\section{Glyphipterigidae}

Glyphipterix haworthana (Stph.). 9, 10, 12, 18, 23, 31, 32, $38-10,43-29,43-25,43-15,43-5,44,55,59 a, 63$, $68,70,73,77$.

\section{Lyonetiidae}

Lyonetia frigidariella H.-S. 37: 1 ex [1899] (Poppius leg.). Lyonetia ledi Wck. 28 (Krogerus 1943).

\section{Oecophoridae}

Semioscopis avellanella (Hb.). 9 (Valle 1933), 10, 29, 48. Agonopterix heracliana (L.). 46 (Znamenskaja 1962). Agonopterix broennoeensis (Benander). 46: 1 ex 5.7.1933 (in ZIN).
Pseudatemelia josephinae (Toll). 5, 9, 10, 27 (Krogerus 1943), 36, 38-15, 61, 64a, 67.

Schiffermuelleria similella (Hb.). 3, 5, 10, 12, 27 (Krogerus 1943), 43-41.

Schiffermuelleria stipella (L.). 2, 3, 5, 6, 8, 9, 10, 12, 15, $17,18,28,42 \mathrm{a}, 43-29,43-23,43-3,44,44 \mathrm{a}, 48,55$, $61,64 a, 65,67,70,71,73,76,77$.

Endrosis sarcitrella (L.). 1, 2, 11, 31, 55.

Pleurota bicostella (Cl.). 2, 4, 5, 6, 8, 9, 12, 15, 18, 23, 28, $34,38-15,38-11,39,40,43-29,43-17,43-16,43-$ $14,43-8,43-3,44,44 a, 46$ (up to $600 \mathrm{~m}$ ), 48, 55, 56, $58 \mathrm{a}, 60,61,64 \mathrm{a}, 65,67,68,70,71,73,76,77$,

\section{Elachistidae}

Elachista kilmunella Stt. 38-14, 38-16, 39, 43-20, 43-5, 44, 55.

Elachista leifi Kaila et Kerppola. 43-5: 5 exx 15.7.1991 (marsh), 55: 10 exx 12-18.7.1991 (marsh, flying at dawn). This recently described species (Kaila \& Kerppola 1992) is so far recorded only from Northern Finland (Kuusamo) and Kola Peninsula.

Elachista parasella Traugott-Olsen. 44a: 1 ex 19.7.1993, 48 (S slope, $700 \mathrm{~m}$ ): 1 ex 15.7.1991.

Elachista alpinella Stt. 55: 1 ex 27.7.1991 (marsh).

Elachista diederichsiella E.Hering. 77: 3 exx 17.7.1992.

Elachista nobilella Z. 43-14: 1 ex 28.6.1991.

Elachista apicipunctella Stt. 55, 56, 58a, 73, 77.

Elachista nielswolffi Svensson. 10: 1 ex 20.7.1979, 43-20: 1 ex 22.7.1992.

Elachista humilis Z. 55: 1 ex 18.7.1991, 58: 1 ex 34.7.1993.

Elachista canapennella (Hb.). 21 (incanella: Valle 1933), 48 (Seitjaur): 1 ex [25.7.1887] (Palmén leg.).

Elachista monosemiella Rössler [=cerusella (Hb.)]. 61 (White Sea coast): 5 exx 5-7.7.1988, 6 exx 2528.7.1991, 70: 2 exx 15.7.1992. A maritime species; the nearest records are from the southern coast of Finland. Elsewhere distributed widely in Central and NW Europe, Russia and Asia Minor (Traugott-Olsen \& Schmidt Nielsen 1977). Species name replaced by Kaila (1992).

Elachista subalbidella Schl. 60: 1 ex [1870] (Sahlberg leg.).

Biselachista eleochariella (Stt.). 55: 1 ex 18.7.1991.

Biselachista albidella (Nylander). 43-9: 1 ex 20.7.1993, 55: 1 ex 18.7.1991.

Cosmiotes exactella (H.-S.). 10, 37, 38-15, 43-1, 48 (Kuftuai, $700 \mathrm{~m}$ ), 58.

Cosmiotes freyerella (Hb.). 39, 43-29, 43-20, 55, 58a, 70.

\section{Coleophoridae}

Coleophora uliginosella Glitz. 55: 1 ex 17.7.1991 (marsh). Coleophora serratella (L.). 46, 48 (Iarval cases on Betula tortuosa), 55, 64a.

Coleophora betulaenanae Klim. 44, 46, 48 (larval cases on Betula nana and B. tortuosa). The status of the 
species is uncertain; it might be conspecific with $C$. vacciniella $\mathrm{H} .-\mathrm{S}$.

Coleophora viminetella Z. 43-3, 61, 64a, 67.

Coleophora unigenella Svensson. 48: 1 ex 15.7.1991. This recently described species (Svensson 1966) has so far only been recorded from northern Sweden, the Kilpisjärvi area in Finland (Jalava 1977) and Central Norway (Opheim \& Fjeldså 1980). Larva mines the leaves of Dryas actopetala (Kyrki \& Karvonen 1984). New for Russia.

Coleophora idaeella Hofm. 9 (Valle 1933), 10, 27 (Krogerus 1943), 43-29, 43-15.

Coleophora vacciniella H.-S. 10 (Sinev 1988), 27 (Krogerus 1943), 28, 38-11, 43-29, 43-15, 44a, 48, 65.

Coleophora ledi Stt. 38-15, 43-15, 43-9, 43-5, 44 (larval case on Ledum palustre), 55.

Coleophora vitisella Gregson. 17 (Valle 1933), 27 (Krogerus 1943).

Coleophora glitzella Hofm. 15 (Valle 1933), 28, 42a, 43$15,44 \mathrm{a}, 48,64 \mathrm{a}, 65$.

Coleophora murinella Tengstr. 27 (Krogerus 1943), 28, 43-29, 43-23, 43-20, 43-15, 44a, 48, 55.

Coleophora thulea Johansson. 34: 1 ex [12-16.7.1887] (Palmén leg.), 61: 1 ex 28.7.1991. The species is described from Swedish Lapland (Johansson 1967) and has so far been recorded only from northern parts of Finland, Norway and Sweden (Gustafsson et al. 1987) and from the Kola Peninsula (Kyrki 1981b). Larva feeds on Rubus chamaemorus (Kyrki op. cit.).

Coleophora obscuripalpella Kanerva. 28 (Krogerus 1943), 60: 1 ex [1870] (Sahlberg leg., paratype).

Coleophora frischella (L.). 48, 55, 56, 61, 64a, 77.

Coleophora antennariella H.-S. 59a: 1 ex 3.7.1993. In Finland northwards to province PPe (Kyrki 1978).

Coleophora adjunctella Hodgkinson. 61, 67 (common on sea shore meadows).

Coleophora glaucicolella Wood. 38-16: 1 ex 27.7.1991, 55: 3 exx 30.6-17.7.1991.

Coleophora murinipennella (Dup.). 58a: 1 ex 4.7.1993, 60: 1 ex [1870] (Sahlberg leg.).

Coleophora alticolella Z. 44a, 55, 61, 67, 70, 73.

Coleophora virgaureae Stt. 6, 9 (Valle 1933), 10, 15, 28, 43-15, 44, 46 (600 m), 48 ( $850 \mathrm{~m}), 55,56,61,70$.

Coleophora boreella (Ben.). 64a: 1 ex 4.7.1993. So far known only from Finland, Sweden and Norway, where it has been recorded from scattered localities throughout the whole area; seems to prefer coastal areas. In $\mathrm{MZH}$, most of the material is from the southern province $\mathrm{Al}$ from sea shore meadows. New for Russia.

Coleophora saxicolella (Duponchel), 9 (Valle 1933), 75: 2 ex 15.7.1992.

Coleophora atriplicis Meyr. 60: 1 ex [1870] (Sahlberg leg.).

Coleophora pappiferella Hofm. 28: 1 ex [1899] (Poppius leg.), 55: 1 ex 5.7.1991.

Coleophora trochilella (Dup.). 55: 1 ex 17.7.1991.

Coleophora striatipennella Nylander. 55: 2 exx 30.613.7.1991, 56: 1 ex 19.7.1991, 58a: 1 ex 4.7.1993.

Coleophora paripennella Z. 10: 1 ex 10.7.1938.

\section{Momphidae}

Mompha locupletella (Den. et Schiff.). 9, 10, 12, 55.

Mompha raschkiella (Z.). 39, 53, 56, 58a, 73.

Mompha complexa Svensson. 10: 11 exx 8-25.6.1980.

Mompha idaei (Z.). 10, 12, 14, 27 (Krogerus 1943), 39, 43-7, 43-3, 43-1, 44, 45, 56, 60, 64a, 67, 70, 71.

Mompha conturbatella (Hb.). 10, 12, 43-15, 43-1, 44, 55, 56.

Mompha lacteella (Stph.). 44, 55, 56, 61.

\section{Scythrididae}

Scythris obscurella (Scop.). 60: 1 ex [17.7.1870] (Sahlberg leg.), 72: 1 ex 15.7.1992.

Scythris fuscopterella Bengtsson. 39: 1 ex 22.7.1992, 72: 7 exx 15.7.1992. Recently described from northern Sweden (Bengtsson 1977). Additionally it has been recorded only from northern Finland (e.g. Laasonen 1980). Bengtsson (pers. comm.) has found it also in a material collected by K.Mikkola in the Chukchi Peninsula. Biology unknown; in the locality 72 moths were collected on the flowers of Leucanthemum vulgare. New for Russia.

\section{Gelechiidae}

Teleiodes paripunctella (Thnbg.). 55, 56, 67, 73.

Teleiodes proximella (Hb.). Lapponia Rossica 1 ex [1829] (Fellman leg.), 55.

Teleiodes epomidella (Tengstr.). 21 (Valle 1933), 27 (Krogerus 1943), 38.

Teleiopsis diffinis (Hw.). 10, 12, 44, 60.

Athrips pruinosellus (Lienig et Z.). 9, 18, 21, 55, 56, 67.

Bryotropha similis (Stt.). 61: 1 ex 25.7.1991, 70: 7 exx 15.7.1992.

Bryotropha boreella (Dougl.). 55 (peat bog, common at dawn in 1991), 56: 1 ex 10.7.1991.

Bryotropha galbanella (Z.). 12, 15, 27 (Krogerus 1943), $44,60,65$.

Bryotropha terrella (Den. et Schiff.). 60: 1 ex [1870] (Sahlberg leg.).

Bryotropha plantariella (Tengstr.). 62: 1 ex 11.8.1987.

Chionodes viduella (F.). 1, 4, 5, 6, 9, 10, 12, 15, 17, 18, 21, $23,27,28,31,38-15,43-17,43-8,44,44 a, 52,53$, $60,67,73$.

Chionodes lugubrella (F.). 12, 21, 27 (Krogerus 1943), 28, 36, 38-15, 39, 43-29, 43-20, 45, 53, 59a, 67.

Chionodes nubilella (Zett.). 10, 12, 14, 21, 27 (Krogerus 1943), 48 (S slope, $700 \mathrm{~m}$ ), 65. The specimens from the locality 65 have light grey forewings due to a mixture of white and dark scales.

Chionodes continuella (Z.). 3, 4, 6, 8, 9, 10, 12, 15, 18, 21, $28,42 \mathrm{a}, 44 \mathrm{a}, 46,48(850 \mathrm{~m}), 56,60,65$.

Chionodes luctuella (Hb.). 43-29: 1 ex 2.8.1993, 43-20: 3 exx 8-22.7.1992 \& 1 ex 25.7.1993, 56a: 1 ex 24.7.1993.

Lita sexpunctella (F.). 9, 12, 17, 18, 23, 28, 29, 31, 37, 3814, 39, 43-29, 44, 46, 48, 58a, 63, 65, 67, 68, 73. 
Aroga velocella (Z.). 60: I ex [1870] (Sahlberg leg.), 67: 1 ex 7.7.1987.

Neofaculta infernella (H.-S.). 5, 6, 8, 9, 10, 12, 15, 18, 21, $23,28,31,38-15,38-11,39,43-29,43-20,43-16$, $43-7,43-1,44$ (up to $700 \mathrm{~m}$ ), $46,48,53,55,56,56 \mathrm{a}$, $60,65,67,73$.

Neofaculta ericetella (Geyer). 28 (Krogerus 1943) (common).

Altenia perspersella (Wck.). 15 (Valle 1933), 18, 28 (Krogerus 1943), 38-11, 39, 42a, 43-29, 43-15, 44 (500 m), 44a, 55, 67.

Gnorimoschema valesiellum (Stgr.). 8 (Valle 1933), 28: 2 exx [1899] (Poppius leg.).

Gnorimoschema nordlandicolellum Strand. 64a: 2 exx 21.7.1993. Distributed in Finland northwards to the province Kb (Kyrki 1978), in Russia recorded from Novgorod (Piskunov 1981). Lives on sandy meadows, even without Thymus (Jalava, unpubl.).

Scrobipalpula psilella (H.-S.). 68: 1 ex 21.6.1985.

Scrobipalpa acuminatella (Sircom). 55: 4 exx 30.613.7.1991, 60: 1 ex 2.7.[1870] (SahIberg leg.).

Scrobipalpa murinella (Dup.). 44a, 48, 55, 56, 68, 73.

Scrobipalpa obsoletella (F.R.). 73 (White Sea shore): 8 exx 25.6.1985.

Scrobipalpa atriplicella (F.R.). 75: 3 exx 15.7.1992.

Sophronia gelidella Nordman. 46 (Kukis-wum-chorr, 900 m): 1 ex 18.7.1986, 56: 1 ex 18.7.1986. This alpine species is so far recorded in northern Sweden, Norway and province Le (Kilpisjärvi, the type locality) in Finland (Gustafsson 1987, Kyrki 1978) and from SW Altai (Exp. Mikkola, Hippa \& Jalava, in ZMH). New for Russia.

Aproaerema anthyllidella (Hb.). 44a (600 m, tundra): 1 ex 14.7.1993.

Aproaerema karvoneni Hackman. 61: 1 ex 30.6.1989, 68: 1 ex 21.6.1985. In Finland distributed northwards to the province Li (Kyrki 1978). In Sweden it is known from 13 provinces from Närke to Lule Lappmark. In Norway it is only recorded from the central part of the country. Larva on Fabaceae (Itämies \& Kyrki 1983). New for Russia.

Acompsia subpunctella Svensson. 70: 1 ex 15.7.1992, 75: 1 ex 15.7.1992. Recently (Svensson 1966) described from Northern Sweden; it has been recorded from Finland (northern parts and south coast), Latvia (Piskunov 1981), Southern Siberia: SW-Altai (Exp. Mikkola, Hippa \& Jalava, in ZMH). Larva feeds on Veronica longifolia (H. Bruun, pers. comm.). New for Russia.

\section{Cossidae}

Cossus cossus L. 27 (Krogerus 1943).

\section{Tortricidae}

Pandemis cerasana (Hb.). 61: 3 exx 7.7.1988.

Argyrotaenia ljungiana (Thnbg.). 28 (Krogerus 1943), 36 (Krogerus 1943), 46 (pheromone traps, 1984).
Choristoneura albaniana (Walker) [= lapponana (Tengstr.)]. 1, 5, 8, 9, 10, 12, 14, 18, 21, 36, 38-8, 38$16,39,42 \mathrm{a}, 43-29,43-23,43-20,43-14,43-12,43-$ $3,44,44 a, 53,60,63,64,65,67,73$. Seems to fly only in odd years. Synonymy established by Dang (1992).

Syndemis musculana (Hb.). 38-15, 38-11, 43-15, 43-41, $46,55,58 \mathrm{a}, 60,61,63,67$.

Aphelia viburnana (Den. et Schiff.). 1, 2, 4, 6, 8, 10, 12, $14,15,26,27,38-16,42 \mathrm{a}, 43-29,44 \mathrm{a}, 46,48,52,55$, $60,65,67,76$.

Clepsis senecionana (Hb.). 38-15, 38-11, 39, 40, 42a, 43$29,43-23,43-20,43-15,44,46,48,53,55,56,56$ a, $58 \mathrm{a}, 59 \mathrm{a}, 60,63,64 \mathrm{a}, 67,73,77$.

Clepsis rogana (Gn.). Lapland 1 ex [no date, coll. Tengström], 34: 2 exx [29.7-9.8.1887] (Palmén leg.), 65: 6 exx [1899] (Montell leg.), 72: 3 exx 15.7.1992, 73: 4 exx 18.7.1992, 75: 9 exx 15.7.1992, 76: 1 ex 15.7.1992, 77: 2 exx 17.7.1992. This species has not been recorded from Fennoscandia before, but it is widespread from Komi region (northern Ural) through Siberia to the Pacific coast, and in most of the mountain systems of Central Europe (Kuznetsov 1978). The polyphagous larva is reported from Vaccinium myrtillus and many marsh plants (e.g. Veratrum, Luzula) (op. cit.).

Lozotaenia forsterana (F.). 3, 4, 6, 8, 9, 10, 12, 18, 21, 23, $28,31,38-13,43-29,43-23,43-14,43-8,44,44 a$, $48,52,55,56,56 a, 59 a, 61,64,64 a, 69,73$.

Philedone gerningana (Den. et Schiff.). 43-29: 1 ex 28.7.1992, 73: 2 exx 18.7.1992.

Eulia ministrana (L.). 3, 5, 6, 9, 10, 12, 15, 21, 23, 28, 31, $34,38-5,38-8,38-11,38-15,39,42 \mathrm{a}, 43-29,43-23$, $43-20,43-17,43-14,43-7,43-3,44,44 a, 45,46,48$, $55,56,58 \mathrm{a}, 59 \mathrm{a}, 60,61,63,64 \mathrm{a}, 65,67,71,77$.

Eana osseana (Scop.). 1, 2, 3, 5, 6, 8, 10, 12, 13, 14, 15, $19,34,39,43-29,43-25,43-20,44,46,49,55,56$, $60,61,65$.

Eana argentana (Cl.). Lapponia Rossica [1870] (Tengström, unpubl; no later confirmation).

Eana penziana (Thnbg.). 73: 1 ex 18.7.1992.

Acleris comariana (Lienig et Z.). 56: 1 ex 19.8.1984. A single specimen collected in Finnish Lapland from the province Li (Koponen et al. 1982).

Acleris implexana ferrumixtana (Benander). 17: 1 ex [1899] (Poppius leg.), 18: 3 exx 18.6.1929, 31: 1 ex [8-29.6. 1887] (Palmén leg.), 62 (sec. Peltonen 1947).

Acleris aspersana (Hb.). 10: 1 ex 27.8.1979.

Acleris fimbriana (Thnbg.). 46 (Fridolin 1936), 52: 1 ex [9-12.8.1861] (Inberg leg.).

Acleris maccana (Tr.). 10, 28, 37.

Trachysmia vulneratana (Zett.). 15: 1 ex 15.7.1931, 55: 1 ex 18.7.1991.

Eupoecilia sanguisorbana (H.-S.). 55: 20 exx 17-18.7.1991 (on a Sanguisorba growing marsh), 60: 2 exx 1730.7.[1870], 75: 1 ex 15.7.1992. The species was not recorded from northern Fennoscandia before, it has been reported from southern Sweden (the island of Gotland) and from southern Norway. Widely distributed in Central Europe (Razowski 1970). The larva feeds in the seed heads of Sanguisorba officinalis, 
which has a disjunct distribution in northwestern Europe: Kola Peninsula, Gotland and southern Norway (Hultén 1971).

Aethes cnicana (Westw.). 70, 75, 76.

Aethes deutschiana (Zett.). 1, 2, 3, 5, 8, 9, 10, 12, 14, 15, $36,42 \mathrm{a}, 43-29,44,44 \mathrm{a}, 46$ (up to Kukis-wum-chorr, $900 \mathrm{~m}$ ), 48 (up to mountain tundra), 55, 65, 67 .

Aethes triangulana (Tr.). 58a: 3 exx 4-6.7.1993, 73: 8 exx 25.6.1985, 77; 1 ex 17.7.1992.

Aethes smeathmanniana (F.). 23, 29, 39, 55, 56, 58a, 64a, $70,73,75,77$.

Aethes rutilana (Hb.). 70: 1 ex 15,7.1992.

Cochylidia subroseana (Hw.). 55, 56, 58a, 67, 70.

Cochylis dubitana (Hb.). 8, 9, 10, 12, 21, 23, 31, 42a, 4323,44 (up to $700 \mathrm{~m}$ ), $46,48,55,56,60,67$.

Sparganothis rubicundana (H.-S.). 2, 3, 4, 5, 6, 8, 9, 10 , $12,15,21,28,34,38-15,38-11,39,42 \mathrm{a}, 43-41,43-$ 20, 43-16, 43-7, 44, 44a, 46, 48, 55, 56, 56a, 60, 61.

Sparganothis abiskoana (Car.). 28 (Krogerus 1943), 46: 2 exx 22-26.7.1926 (in ZIN) \& 1 ex 31.7.1993, 48: 2 exx 11.7.1986 \& 1 ex 15.7.1991.

Celypha cespitana (Hb.). 12 (Menikkajoki) (Valle 1933), 44: 1 ex 30.7.1981.

Olethreutes ledianus (L.). 6, 8, 9, 10, 12, 18, 21, 28, 34, $38-13,42 \mathrm{a}, 43-41,43-29,43-18,43-14,43-8,44$, $44 \mathrm{a}, 48,53,55,60,64 \mathrm{a}$.

Olethreutes obsoletanus (Zett.). 1, 2, 5, 8, 9, 10, 12, 13, 15, $17,21,25,27,28,34,38-15,43-29,44$ (up to $800 \mathrm{~m}$ ), $44 \mathrm{a}, 45,46$ (up to $600 \mathrm{~m}$ ), $48,51,55,56 \mathrm{a}, 60,61,65$, $67,69,71$.

Olethreutes dissolutanus (Stange). 27 (Krogerus 1943), 46, 61.

Olethreutes mygindianus (Den. et Schiff.). 28 (Krogerus 1943), 38-15, 38-11, 43-29, 43-20, 43-14, 43-12, 43-8, 43-3, 67.

Olethreutes arbutellus (L.). 43-15, 43-9, 44, 44a, 52, 73, 77.

Olethreutes aquilonanus (Karvonen), 42a: 3 exx 1218.7.1993, 48 (Ninchurt, 900 m): 1 ex 9.7.1986.

Olethreutes noricanus (H.-S.). 12 (Nurmensätti): $1 \mathrm{cx}$ 15.6.1937, 42a: 1 ex 12.7.1993, 46 (Kukis-wum-chorr, $900 \mathrm{~m}): 2$ exx 14-18.7.1986 \& (Loparskij, $600 \mathrm{~m}$ ): 1 ex 27.7.1991, 48: 1 ex 15.7.1991. The easternmost records of this species, which is distributed in the Scandinavian mountains and the Alps.

Olethreutes lacunanus (Den. et Schiff.). 1, 2, 3, 6, 8, 9, 10, $12,13,14,15,18,28,36,38-15,46,48,55,56,58 \mathrm{a}$, $60,61,62,64 a, 65,67,69,70,75,77$.

Olethreutes bipunctanus (F.). 1, 2, 3, 5, 6, 8, 9, 10, 12, 15, $18,21,28,34,38-15,43-29,43-14,44$ (up to $800 \mathrm{~m}$ ), 44a, 46 (up to Kukis-wum-chorr, 900 m), 60, 61, 64a, $67,70,73$.

Olethreutes hyperboreanus (Karvonen). 8, 12, 15, 23, 27 , $28,43-41,44 a, 46,48$.

Olethreutes olivanus (Tr.). 44, 48, 55.

Olethreutes palustranus (Lienig et Z.). 3, 4, 8, 10, 12, 15, $51,56 \mathrm{a}, 60,69,76$.

Olethreutes metallicanus (Hb.). 5, 9, 12, 17, 21, 28, 64, 65, $67,69,76$.
Olethreutes schulzianus (F.). 1, 2, 3, 5, 6, 8, 9, 10, 12, 15, $18,21,23,28,31,34,38-15,38-11,39,42 \mathrm{a}, 43-29$, $43-23,43-20,43-16,43-14,43-7,44,44 a, 46$ (up to Kukis-wum-chorr, $900 \mathrm{~m}), 48,51,53,55,56,56 \mathrm{a}$, $58 \mathrm{a}, 60,61,64 \mathrm{a}, 65,67,69,71,76,77$.

Olethreutes schaefferanus (H.-S.). 2, 3, 6, 8, 10, 12, 13, 15 , $18,21,23,34,43-9,44,44 a, 46$ (up to Kukis-wumchorr, $900 \mathrm{~m}), 48,60,65$.

Olethreutes turfosanus (H.-S.). 4, 5, 6, 8, 9, 12, 28, 38-15, 38-11, 42a, 43-29, 43-20, 43-15, 44, 44a, 46 (Kukiswum-chorr, $900 \mathrm{~m}$ ), 48 (subalpine birch forest), 52 , $58 \mathrm{a}, 60,63,73$.

Olethreutes concretanus (Wck.). 9, 15, 18, 42a, 43-20, 44, 44a, 46, 48.

Olethreutes rivulanus (Scop.). 9, 18, 21 (Valle 1933), 43$15,43-9,43-3$.

Pseudohermenias abietana (F.). 48 (forest zone): 1 ex 6.7.1986, 58a: 1 ex 4.7.1993.

Hedya atropunctana (Zett.). 12, 18, 28, 38-8, 38-13, 39, $43-20,43-16,43-7,44,53,61,64 \mathrm{a}, 68,73,77$.

Hedya roseomaculana (H.-S.). 1, 9, 43-29, 43-23, 43-20.

Orthotaenia undulana (Den. et Schiff.). 17, 28, 38-16, $43-29,43-20,44,45,46,55,56,56 a, 61,64 a$.

Apotomis infida (Hein.). 5, 9, 43-3, 61, 64a.

Apotomis moestana (Wck.). 3, 6, 9, 10, 12, 18, 21, 28, 37 . $39,55$.

Apotomis boreana Krogerus. 8, 12, 15, 18, 28, 38-2, 38$13,39,42 \mathrm{a}, 43-23,43-14,43-12,43-7,43-3,44$ (up to $650 \mathrm{~m}), 44 \mathrm{a}, 52,56,64 \mathrm{a}, 73$.

Apotomis sororculana (Zett.). 8, 9, 10, 12, 15, 23, 28, 34, $36,39,43-41,43-17,43-12,46,60,73$.

Apotomis lemniscatana (Kenn.). 10, 12, 14, 34, 38-15, $42 \mathrm{a}, 44 \mathrm{a}, 77$.

Apotomis algidana Krogerus. 9, 18, 38-16, 38-3, 39, 4341, 43-9, 43-5, 44a, 46, 53.

Apotomis sauciana (Fröl.). 1, 2, 3, 4, 8, 9, 12, 15, 18, 38-3, $38-10,39,40,42 a, 43-29,43-25,43-20,43-15,43-$ $5,44,44 a, 45,46,48,53,56 a, 64 a$.

Apotomis demissana (Kenn.). 1, 2, 4, 8, 10, 12, 15.

Endothenia marginana (Haw.). 55: 5 exx 17-18.7.1991, 61: 1 ex 30.6.1989 \& 1 ex 25.7.1991.

Bactra lancealana (Hb.). 27 (Krogerus 1943), 46, 55, 60, $61,64 a, 65,70$.

Ancylis laetana (F.). 67: 1 ex [1885] (Edgren leg.).

Ancylis comptana (Fröl.). 9, 18, 21, 27, 28, 37, 38-16, 43$15,59 \mathrm{a}, 60,65,66,73$.

Ancylis uncella (Den. et Schiff.). 9, 10, 12, 18, 23, 28, 31, $38-13,38-14,39,43-29,43-25,43-19,43-12,43-3$, $44,46,48,53,56,56 \mathrm{a}, 58 \mathrm{a}, 59 \mathrm{a}, 61,63,67,73,77$.

Ancylis unguicella (L.). 6, 8, 9, 10, 12, 15, 18, 21, 23, 28, $29,31,32,37,38-20,38-11,39,40,42 \mathrm{a}, 43-29,43-$ $20,43-15,43-8,44,44 a, 51,53,55,56,56 a, 58 a, 60$, $63,64,64 a, 65,66,67,68,69,73,77$.

Ancylis geminana (Don.). 12, 55, 65, 67, 73, 77.

Ancylis subarcuana (Douglas). 12 (Valle 1933), 18, 37 , 43-14, 53, 55, 58a, 64a, 65, 67, 68, 73.

Ancylis badiana (Den. et Schiff.). 55, 56, 58a, 61, 64a, 67, $70,73,77$. 
Ancylis tineana (Hb.). 56: 1 ex 13.7.1991, 73: 2 exx 25.7.1985.

Ancylis myrtillana (Tr.). 2, 4, 5, 6, 8, 9, 10, 12, 17, 18, 21, $23,28,29,31,38-15,38-3,39,40,42 \mathrm{a}, 43-29,43-$ $25,43-23,43-20,43-12,43-3,44,44 a, 46$ (up to Kukis-wum-chorr, $800 \mathrm{~m}$ ), 48 (up to $900 \mathrm{~m}$ ), 51, 52, $53,55,56,56 a, 58 a, 59,60,61,63,64 a, 65,67,68,70$, $71,73,75,77$.

Epinotia trigonella (L.). 11: 1 ex 15.8.1979.

Epinotia indecorana (Zett.). 18 (Valle 1933).

Epinotia subocellana (Don.). 27 (Krogerus 1943), 43-20, $55,64 \mathrm{a}, 73,75,77$.

Epinotia tetraquetrana (Hw.). 3, 5, 6, 8, 9, 10, 12, 15, 18, 21, $23,29,32,38-14,38-3,39,40,42 a, 43-29,43-25,43-$ $16,43-14,43-8,44,44 a, 46,48,53,55,56,58 a, 60$, $64 a, 67,68,70,73,75,77$.

Epinotia nemorivaga (Tengstr.). 42a: 2 exx 18-23.7.1993, 43-9: 1 ex 11.7.1993, 44a: 7 exx 15-19.7.1993, 48: 2 exx 14.7.1991.

Epinotia tedella (C1.). 38-15, 44, 46, 55, 61, 64, 67, 73, 75. Epinotia cruciana (L.). 2, 5, 6, 8, 9, 10, 12, 13, 15, 18, 19 , $23,28,31,32,38-11,39,43-12,43-2,44,46,55,56$, $60,64 a, 77$.

Epinotia mercuriana (Fröl.). 15, 19, 24, 44.

Epinotia gimmerthaliana (Lienig et Z.). 23 (Djakonov 1911), 60: 1 ex 19.7.1986.

Epinotia nanana (Tr.). 38-14, 39, 42a, 43-29, 43-20, 4317, 44 (up to $600 \mathrm{~m}$ ), 46,48 (up to subalpine birch forest), 55, 56, 56a, 60, 61 .

Epinotia crenana (Hb.). 10: 1 ex 25.5.1980.

Rhopobota ustomaculana (Curt.). 61: 2 exx 25.7.1991.

Rhopobota naevana (Hb.). 23, 43-16, 44, 62.

Zeiraphera griseana (Hb.). 43-29, 44 (common in 1980) (Kozlov 1981b), 46 (Fridolin 1936).

Gypsonoma nitidulana (Lienig et Z.). 5, 8, 9, 10, 12, 15, $18,21,28,38-16,39,43-29,43-25,43-3,44,44 a, 46$ (up to $600 \mathrm{~m}$ ), 48 (up to $900 \mathrm{~m}$ ), 56, 63, 65, 67, 77 .

Epiblema cynosbatella (L.). 73: 1 ex 18.7.1992.

Epiblema sticticana (F.). 56: 1 ex 13.7.1991.

Epiblema cirsiana (Z.). 55: 5 exx 30.6.1991 \& 4 exx 18.7.1991.

Epiblema simploniana (Dup.). 46, 48 (600-800 m) (common in Salix bushes).

Eriopsela quadrana (Hb.). 2, 3, 6, 8, 9, 10, 12, 15, 18, 21, $23,28,31,34,38-11,39,44,44 a, 46,48$ (up to 900 m), 56, 58a, 59a, 63, 65, 67, 68, 73, 77 .

Eucosma obumbratana (Lienig et Z.). 61: 1 ex 29.7.1991, 64a: 2 exx 21.7.1993, 70: 2 exx 15.7.1992, 75: 1 ex 15.7.1992.

Eucosma suomiana (Hoffm.). 75: 2 exx 15.7.1992. In Fennoscandia this rare species has been recorded from central and northern Sweden (provinces Sö, Upl, Nb) (Gustafsson 1987). In Finland it has a few populations on the southern coast (provinces $\mathrm{Ab}, \mathrm{N}, \mathrm{St}$ ), in the northern part it has been recorded from the provinces Ob, Ks and LKem (Kyrki 1978). Kuznetsov (1978) reported it also from northern, northwestern and central parts of Russia. The larva feeds on Solidago.
Eucosma aspidiscana (Hb.). 10, 12, 18, 44, 46, 48, 55, 56, 58a, 59a, 60, 67, 68, 73, 77.

Blastesthia posticana (Zett.). 12 (Leukakoski) (Krogerus 1943), 67: 1 ex 7.7.1987.

Retinia resinella (L.). 12, 18, 23, 28, 40, 43-7, 44, 46, 48, $51,55,60,61,67,68$.

Pammene clanculana (Tengstr.). 1, 2, 9, 10, 12, 15, 18, 21, $23,28,37,43-8,44 \mathrm{a}, 46,60$.

Pammene aurana (F.). 61, 64a. Surprisingly, quite common (tens of specimens) in 1988-1991 on a single small $\left(\approx 200 \mathrm{~m}^{2}\right)$ forest meadow of Pastinaca near 61 . In Fennoscandia this southern species is previously recorded from Russian Karelia near the lake Onega, from southernmost Sweden (Sk) (Gustafsson 1987) and from southwestern Finland (A1, Ab) (Kyrki 1978).

Cydia pactolana (Z.). 43-29: 2 exx 25.7-2.8.1993, 43-5: 2 exx 11-27.7.1993, 45: 1 ex 12.7.1991, 61: 1 ex 25.7.1991.

Cydia cosmophorana (Tr.). 9 (Valle 1933), 21, 28 (Valle 1933), 55.

Cydia coniferana (Sax.). 27 (Krogerus 1943).

Cydia strobilella (L.). 43-29, 44, 48, 58a.

Cydia orobana (Tr.). 44, 60, 61, 64a, 70, 73, 75.

Cydia illutana (H.-S.). 73: 3 exx 25.6.1985.

Cydia aureolana (Tengstr.). 1, 9, 15, 28, 42a, 44, 46, 48, 73,77 .

Cydia compositella (F.). 55, 56 (common in June, 1991, in both sites), $58 \mathrm{a}$.

Dichrorampha flavidorsana Knaggs. 61: 3 exx 28.7.1991.

Dichrorampha obscuratana (Wolff). 61, 70, 73. Very abundant on meadows on Tanacetum.

Dichrorampha cinerascens (Danil.). 70, 72, 73, 75, 76. Common on sea shore meadows. In Fennoscandia recorded only from the southern coast of Finland. Elsewhere distributed from central Europe to western and southern Siberia (Altai) (Kuznetsov \& Jalava 1988). Larva on Achillea.

Dichrorampha plumbana (Scop.). 58a: 10 exx 3-6.7.1993. Previous record from 73 (Kozlov 1977) was based on erroneously determined specimens of $D$. obscuratana (Wolff).

Dichrorampha sedatana (Busck). 72: 1 ex 15.7.1992, 77: 1 ex 17.7.1992. Larva on Tanacetum which is abundant along the White Sea coast.

\section{Choreutidae}

Anthophila fabriciana (L.). 23, 39, 56, 61, 67.

\section{Sesiidae}

Sesia bembeciformis (Hb.). 43-9: 2 exx 27.7.1993. Quite surprizingly found in the industrial wastelands close to "Severonikel" smelter. Larva boring in stems of Salix caprea. Only a few records are known from Fennoscandia: Sweden - one record from province Sk in 1926; Norway - one record from province ST in 
1971; Finland - four old $(1883,1913,1935,1944)$ records from the provinces Sb and LkW (Vuola \& Korpela 1977). In Northern Russia, a single specimen (kept in ZIN) has been collected near Petrozavodsk by Günter. So it is probably distributed all over the area, but mostly overlooked as is the general case in the family Sesiidae.

Synanthedon scoliaeformis (Borkh.). 43-41: 1 ex 7.8.1993 (bait-trap).

Synanthedon formicaeformis (Esp.). 43-8: 1 ex 28.6.1989.

Synanthedon culiciformis (L.). 9: 1 ex 12.7.1928.

Synanthedon spheciformis (Den. et Schiff.). 27 (Krogerus 1943).

\section{Schreckensteiniidae}

Schreckensteinia festaliella (Hb.). 9, 12, 23, 27, 28, 31, $43-41,43-15,44,46,48,56,56 a, 60,61,63,68,73$.

\section{Epermeniidae}

Phaulernis fulviguttella (Z.). 2, 5, 10, 12, 13, 15, 43-7, 46, $48,55,56$.

Epermenia chaerophyllella (Goeze). 55: 1 ex 2.6.1993, 61: 4 exx 7-12.8.1991 (mines on leaves of Anthriscus silvestris).

\section{Pterophoridae}

Amblyptilia punctidactyla (Hw.). 12, 28, 37, 43-23, 46, $48,55, .58 \mathrm{a}, 67,68,70,73,77$.

Platyptilia tesseradactyla (L.). 44, 46, 48, 49, 56, 58 a.

Platyptilia calodactyla (Den. et Schiff.). 3, 5, 6, 8, 9, 10, $12,13,15,18,21,28,34,43-29,43-5,44,46,48,55$, $56,60,61,65,66,70,75$.

Platyptilia pallidactyla (Hw.). 39, 44, 55, 56, 61, 70, 73.

Stenoptilia islandica (Stgr.). 15: 4 exx 14.7-5.8.1929.

Pterophorus leucodactylus (Den. et Schiff.). 60: 2 exx [1870] (Sahlberg leg.), 73: 1 ex 19.7.1992.

Leioptilus osteodactylus (Z.). 8, 19, 28, 43-29, 43-23, 43$15,43-7,46,48,55,56,61,67,70,75$.

Leioptilus tephradactylus (Hb.). 3, 4, 5, 6, 8, 9, 10, 12, 14, $21,34,43-29,43-23,44,46,56,60,64 a, 65$.

\section{Pyralidae}

Anerastia lotella (Hb.). 75: 1 ex 15.7.1992, 77: 1 ex 17.7.1992.

Cryptoblabes bistriga (Hw.). 27 (Krogerus 1943).

Polopeustis altensis (Wck.). 2, 8, 15 (Valle 1933), 42a, 46, $48,65,75,77$.

Dioryctria abietella (Den. et Schiff.). 43-29: 1 ex 48.8.1992 (bait-trap).

Pyla fusca (Hw.). 9, 10, 12, 18, 21, 28, 38-15, 43-29, 4320, 43-16, 43-14, 43-7, 43-3, 44, 44a, 46, 72, 75, 77.

Catastia kistrandella Opheim. 46 (Kukis-wum-chorr, 900 m): 1 ex 4.7.1989, 48 (N slope, 700 m): 1 ex 15.7.1991. This species was described in 1963 from Northern Nor- way and has additionally been reported only from the Kilpisjärvi area in the northwestern comer of Finland (Kyrki 1978) and in the province To in North Sweden (Gustafsson 1987). K.Mikkola collected tens of specimens in northeastern Siberia, Upper Kolyma area (in MZH). The larva probably feeds on Arnica angustifolia, because the moth has always been caught flying on dry fell meadows with growths of Arnica. New for Russia.

Myelopsis tetricella (Den. et Schiff.). 8, 9, 10, 12 (Valle 1933), 27 (Krogerus 1943), 44a.

Apomyelois bistriatellus (Hulst). 43-5: 1 ex 23.7.1993, 76: 1 ex 15.7.1992.

Assara terebrella (Zinck.). 43-29: 2 exx 23.7.1992 (baittrap).

Plodia interpunctella (Hb.). 10, 14 (synantropic).

Hypsopygia costalis (F.). 39: 1 ex 23.7.1993 (bait-trap). A southern species, nearest records from the southern coast of Finland (Kyrki 1978). Most probably a migrated specimen.

Pyralis lieniginalis (Z.). 67: 1 ex 7.7.1987.

Chrysoteuchia culmella (L.). 28 (Krogerus 1943).

Crambus ericellus (Hb.). 8, 10, 12, 65, 70, 75, 76.

Crambus alienellus (Germar et Kaulfuss). 9, 12, 18, 27 , 28, 36, 44, 58a, 60.

Crambus pratellus (L.). 10, 15, 44, 55, 56, 60, 64a, 67, 70.

Crambus lathoniellus (Zinck.). 5, 9, 10, 12, 18, 28, 58a, $60,61,64,64 a, 67,69,70,73,75,76,77$

Crambus hamellus (Thnbg.). 27 (Krogerus 1943), 44: 1 ex 30.7.1980, 64a: 1 ex 20.7.1993.

Crambus perlellus (Scop.). 73, 75, 77.

Agriphila straminella (Den. et Schiff.). 60, 61, 65, 77.

Agriphila biarmica (Tengstr.). 28 (Krogerus 1943), 44: 2 exx 29-30.7.1980, 44a: 1 ex 15.7.1993.

Catoptria furcatella (Zett.). 1, 12, 13, 15, 44a, 46, 48.

Catoptria permutatella (H.-S.). 46: 1 ex 17.7.1982, 56: 2 exx 20.7.1986, 64a: 1 ex 20.7.1993.

Catoptria margaritella (Den. et Schiff.). 55: 6 exx 1726.7.1991, 61: 1 ex 28.7.1991.

Catoptria maculalis (Zett.). 3, 8, 9, 10, 12, 17, 18, 27, 4329, 48, 56, 56a, 58a.

Pediasia truncatella (Zett.). 27 (Krogerus 1943), 44: 1 ex 19.7.1981, 58a: 2 exx 4-6.7.1993.

Gesneria centuriella (Den. et Schiff.). 5, 8, 10, 12, 27, 28, $38-3,38-15,39,43-15,43-7,44,46,56$.

Eudonia alpina (Curt.). 2, 5, 8, 9, 10, 15, 18, 21, 28, 31, 34, $36,58 \mathrm{a}$.

Eudonia murana (Curt.). 8, 10, 12, 15, 17, 18, 28, 44, 44a, $46,48,56,60,64 a, 65,66$.

Eudonia sudetica (Z.). 3, 5, 15, 23, 27, 44, 46, 55, 56, 56a, $61,64 \mathrm{a}$.

Titanio schrankiana (Hochenw.). 44: 1 ex 17.7.1981, 48: 3 exx 15.7.1991.

Pyrausta porphyralis (Den. et Schiff.). 12, 28, 48, 52, 55, $56,72,73,77$.

Loxostege sticticalis (L.). 15 (Valle 1933), 46 (Kukis-wumchorr, $900 \mathrm{~m}$ ): 1 ex 18.7.1986. A migrant.

Laxostege commixtalis (Walker). 36 (Krogerus 1943), 45: 1 ex [8.7.1870] (Sahlberg leg.).

Mutuuraia terrealis (Tr.). 56a, 72, 73, 77. 
Anania funebris (Ström). 28 (Krogerus 1943), 44a, 48, 53, $56,58 \mathrm{a}, 59,63,64 \mathrm{a}, 67,70,72,77$.

Opsibotys fuscalis (Den. et Schiff.). 55, 56, 60, 61, 64a, 70, 75.

Udea lutealis (Hb.). 44, 48, 56, 60, 67, 77.

Udea hamalis (Thnbg.). 61: 2 exx 25-28.7.1991.

Udea inquinatalis (Lienig et Z.). 1, 2, 4, 5, 8, 9, 10, 12, 15, $18,21,28,31,34,36,38-16,39,42 \mathrm{a}, 43-29,43-23$, 43-17, 43-14, 43-3, 44 (up to $600 \mathrm{~m}$ ), 44a, 46, 48, 51, $53,55,56,56 \mathrm{a}, 59 \mathrm{a}, 60,64 \mathrm{a}, 67$.

Udea decrepitalis (H.-S.). 1, 2, 4, 5, 8, 9, 10, 12, 13, 15, 18, $21,23,34,43-29,43-14,46,48,55,56,58 a, 65,73,77$.

\section{Zygaenidae}

Zygaena exulans (Hochenw.). 1, 2, 4, 6, 8, 9, 10, 12, 14 , $15,16,18,19,23,28,34,36,44,44 a, 46,48,65$.

\section{Hesperiidae}

Pyrgus andromedae (Wallengr.). 46, 48 (alpine zone).

Pyrgus centaureae (Rambur). 5, 6, 8, 9, 10, 12, 18, 21, 22, $23,28,30,31,36,59,60,67,73$.

Carterocephalus palaemon (Pallas). 23, 43-29, 45, 52, 63. Carterocephalys silvicola (Meig). 59 (Nordström et al. 1955), 60.

Hesperia comma catena (Stgr.). 73, 76, 77, 78, 79.

\section{Papilionidae}

Papilio machaon L. 4, 8, 12, 15, 23, 28, 30, 44, 46, 60, 61 .

\section{Pieridae}

Leptidea sinapis (L.). 12 (Linnaluoto \& Koponen 1980). Pieris brassicae (L.). 9, 12, 14, 15, 44.

Pieris rapae (L.). 10, 12, 21, 23, 44, 45, 60, 63, 75, 77.

Pieris napi (L.). 4, 9, 10, 12, 13, 14, 15, 19, 23, 28, 30, 31, $34,38-3,39,44,46,47,48,51,52,53,54,55,56,58$ a, $60,61,63,64,67,68,69,71,73,77$.

Anthocharis cardamines (L.). 10: 1 ex 25.6.1980, 44: 2 exx 10-17.07.1981, 58a (abundant in 1993).

Colias palaeno (L.). 4, 5, 6, 8, 9, 10, 12, 18, 19, 22, 23, 27, $28,30,31,37,38-13,38-16,43-29,43-23,43-21$, $44,46,47,48,49,51,52,55,56,60,61,64 \mathrm{a}, 67,68$, $69,71,73$.

\section{Lycaenidae}

Callophrys rubi (L.). 4, 12, 23, 28, 30, 40, 43-29, 44, 45, $46,58 \mathrm{a}, 59,59 \mathrm{a}, 64 \mathrm{a}$.

Lycaena phlaeas (L.). 2, 4, 8, 10, 12, 13, 15, 44, 46, 55, 56, $60,61,63,67,68,73,77$.

Lycaena helle (Den. et Schiff.). 8, 9, 10, 12, 45, 59, 60, 61, $62,63,66,67$.

Heodes virgaureae (L.). Lapponia rossica 2 exx [1861] (Inberg leg., in MZH). The only records from the Kola
Peninsula. Since there are no later records of the species, we suppose that the specimens originated from the southernmost part of the area under study, i.e. between Knjazhaja Guba and Kandalaksha (see Silfverberg 1988).

Palaeochrysophanus hippothoe (L.). 30, 53, 59, 60, 63, 64a, 66, 71, 72, 73, 77.

Celastrina argiolus (L.). 4, 15, 59 (Nordström et al. 1955).

Glaucopsyche alexis (Poda). 4 (Nordström et al. 1955).

Lycaeides idas (L.). 9, 12, 14, 18, 28, 38-3, 38-6, 38-10, $38-13,38-16,39,40,43-41,43-29,43-15,46,55$, $56 \mathrm{a}, 61,77$.

Eumedonia eumedon (Esp.). 59 (Nordström et al. 1955). The closest confirmed record in Russia is from Northern Karelia (Srednij island, close to Chupa) (Kozlov, pers.obs.).

Vacciniina optilete (Knoch). 4, 5, 6, 8, 9, 10, 12, 13, 14, $15,18,22,23,28,30,31,34,38-6,38-10,38-13,38-$ $16,39,40,42 \mathrm{a}, 43-29,43-23,43-20,43-16,43-15$, $43-9,43-5,44,44 a, 45,46,48,51,52,53,55,56,56 a$, $60,61,63,65,67,69,71,73,77$.

Agriades glandon aquilo (Boisd.). 3 (Valle 1933), 12 (Sotavalta, unpubl.), 15 (common in June), 46 (Fridolin 1936).

Cyaniris semiargus (Rott.). 30, 44, 46, 52, 55, 56, 60, 61.

Polyommatus icarus (Rott.). 12, 30, 39, 43-15, 55, 56, 60, 61.

\section{Nymphalidae}

Nymphalis antiopa (L.). 9, 12, 19 (Valle 1933), 44, 46, $59 \mathrm{a}$.

Vanessa atalanta (L.). 23: 1 ex August 1973 (V.Isakov, pers. comm.). A migrant.

Vanessa cardui (L.). 7 (Nordström et al. 1955), 10 (Sotavalta, unpubl.), 12 (Valle 1933), 19, 46 (Kukis-wumchorr, $900 \mathrm{~m})$. A migrant.

Aglais urticae (L.). 2, 4, 9, 10, 12, 14, 15, 19, 23, 30, 44, $46,55,56,58 \mathrm{a}, 60,61,63,67$.

Speyeria aglaja (L.). 8, 9, 10, 12, 28, 45, 46, $52,54$.

Boloria napaea (Hoffm.). 1, 2, 12, 14, 15, 31, 46.

Boloria aquilonaris (Stich.). 4, 5, 6, 8, 9, 10, 12, 14, 15, $17,18,19,22,23,30,31,33,34,37,38-16,43-29$, $43-20,44,46,47,49,50,52,55,56,57,60,65$.

Proclossiana eunomia (Esp.). 4, 5, 6, 8, 9, 10, 12, 15, 17, $18,22,23,28,30,31,34,35,36,37,38-15,43-29$, $45,46,52,53,56,60,63,67,68,69,71$.

Clossiana selene (Den. et Schiff.). 4, 5, 8, 9, 10, 12, 14, 15, $18,23,27,28,30,31,37,40,43-29,43-21,44,46$, $48,53,55,61,66$.

Clossiana freija (Thnbg.). 3, 4, 5, 6, 8, 9, 10, 12, 18, 21, $22,23,27,28,29,30,31,34,36,37,42 a, 43-29,43-$ $21,44,44 a, 46,52,53,56,60,65,69,77$.

Clossiana polaris (Boisd.). 4, 8, 12, 15, 22, 28, 36, 44, 46, $48,52,53,65$.

Clossiana thore (Hb.). 8, 9, 10, 12, 23, 29, 31, 60.

Clossiana frigga (Thnbg.). 4, 5, 9, 10, 12, 14, 15, 18, 22, $23,27,30,31,36,37,43-29,44,45,46,52,53,54$, 55,60 . 
Clossiana euphrosyne (L.). 5, 6, 8, 9, 10, 12, 17, 18, 21, 22, 23, 26 (Petersen 1947), 27, 28, 31, 34, 36, 37, 38$10,38-13,38-16,39,40,43-29,43-21,43-16,43-9$, $44,45,46,47,48,52,53,54,60,61,62,63,66,67$, $68,71,73,77$.

Clossiana chariclea (Schn.). 12, 14, 15, 23, 29, 31.

Mellicta athalia (Rott.). 8, 9, 10, 12, 19, 21, 73.

Hypodryas iduna (Dalm.). 4, 5, 8, 10, 12, 15, 23, 34, 35, $36,44(600 \mathrm{~m}), 45,46(600-900 \mathrm{~m}), 48(700-900 \mathrm{~m})$, 52.

\section{Satyridae}

Erebia ligea (L.). 5, 6, 8, 10, 12, 23, 31, 40, 43-41, 44, $44 a, 46,55,56,60,61,63,68,73$.

Erebia disa (Thnbg.). 3, 4, 5, 6, 8, 9, 10, 12, 18, 21, 22, 23, $28,30,31,36,39,42 \mathrm{a}, 44,45,46,48,52,58 \mathrm{a}, 59 \mathrm{a}, 60$, 63,77 .

Erebia embla (Thnbg.). 6, 8, 9, 12, 18, 21, 23, 28, 30, 36, $44,59 \mathrm{a}, 60,63,66,67$.

Erebia pandrose (Bkh.). 1, 2, 3, 4, 5, 8, 9, 10, 12, 15, 19, $22,23,25,28,29,31,34,35,36,42 \mathrm{a}, 43-22,44,44 \mathrm{a}$, $45,46,48,65,73$.

Oeneis norna (Thnbg.). 4, 5, 8, 9, 10, 12, 14, 18, 21, 22, $23,30,36,38-13,43-29,43-23,44,46$.

Oeneis bore (Schn.). 4, 8, 10, 12, 14, 15, 29, 31, 36, 42a, $44,44 a, 45,46$.

Oeneis jutta (Hb.). 30 (V.Isakov, pers. comm.), 66 (Nordström et al. 1955).

Coenonympha tullia (Müll.). 23, 39, 43-21, 44a, 46, 55, $56,61,64 a$.

Iasiommata petropolitana (F.). 59 (Nordström et al. 1955), $63,69$.

\section{Drepanidae}

Falcaria lacertinaria (L.). 8, 9, 10, 12, 14, 15, 18, 21, 28, $37,46,55,58 \mathrm{a}, 60,63,64,67,73$.

Drepana falcataria (L.). 43-20: 2 exx 10-25.7.1993, 55: 1 ex 5.7.1991.

\section{Thyatiridae}

Ochropacha duplaris (L.). 43-35: 1 ex 15.6.1993, 55: 4 exx 29.6-2.7.1991, 60: 1 ex 5.7.1987.

Achlya flavicornis (L.). 9 (Valle 1933), 10, 12, 17, 46 (Fridolin 1936).

\section{Geometridae}

Archiearis parthenias (L.). 8, 9, 10, 12, 44.

Geometra papilionaria (L.). 18 (Valle 1933), 28 (Valle 1933), 46: 1 ex 2.8.1990, 64a: 5 exx 21.7.1993.

Jodis putata (L.). 28 (Krogerus 1943), 45, 48, 58a, 61, 64a, $67,68,70,77$.

Cyclophora albipunctata (Hufn.). 9, 10, 12, 18, 28, 38-15, 43-15, 48 (700 m), 65.

Scopula ternata (Schrank). 5, 6, 8, 9, 10, 12, 13, 17, 18, 21, $23,28,31,34,37,38-10,38-16,43-29,43-23,43-$
$16,43-7,43-3,44,44 a, 46,48,55,56,56 a, 60,61,64$, 64a, 65, 69, 70, 71, 76 .

Scopula frigidaria schoyeni (Schn.). 8, 12 (Valle 1933), 17, 27 (Krogerus 1943), 28, 43-29, 43-20, 43-15, 52, 56 a.

Xanthorhoe designata (Hufn.). 55, 56, 65, 71.

Xanthorhoe abrasaria (H.-S.). 3, 5, 6, 8, 9, 10, 12, 19, 27 , $31,34,36,38-15,43-20,43-15,44,53,55,56,69$, 77.

Xanthorhoe munitata (Hb.). 1, 2, 3, 4, 5, 6, 8, 9, 10, 12, 13, $15,18,23,29,30,31,34,39,43-29,43-23,44,48$, $49,55,56,60,61,62,65,70,73,75,77$.

Xanthorhoe spadicearia (Den. et Schiff.). 1, 2, 3, 6, 9, 12 , 42a, 43-20, 44, 55, 56, 58a, 59a, 63, 64a, 65, 73.

Xanthorhoe ferrugata (C1.). 2, 3, 6, 8, 9, 10, 12, 15, 18, 21, $23,28,36,39,43-15,48,55,56,63,65,66,73$.

Xanthorhoe montanata (Den. et Schiff.). 5, 6, 9, 10, 12, 19.

Xanthorhoe fluctuata (L.). 1, 2, 4, 10, 12, 13, 14, 15, 23, 31.

Xanthorhoe annotinata (Zett.). 1, 2, 4, 5, 6, 8, 9, 10, 12, 15, $18,21,23,28,29,31,34,40,42 \mathrm{a}, 43-29,43-23,44$, $48,52,59 \mathrm{a}, 60,64,67,69,71,73,77$.

Epirrhoe tristata (L.). 17: 1 ex (in MZH) labelled "Pasvik coll. Tengström" without any date and number. The general distribution in Finland extends to the southern part of the province Lkem; the only northern record is from Ivalo (Mikkola et al. 1985). We include this species although it might be collected with equal probability from the Norwegian side of the Patsjoki river.

Epirrhoe hastulata (Hb.). 28: 1 ex [1939] (Platonoff leg.), 64: 1 ex [12-27.7.1885] (Edgren leg.).

Epirrhoe alternata (Müll.). 9, 10, 12, 18, 21, 37, 43-41, $55,56,58 \mathrm{a}, 61,65,66,75$.

Entephria polata (Dup.). 1, 2, 4, 6, 8, 10, 12, 13, 15, 31 (Miller 1915), 42a, 44a, 46, 65.

Entephria byssata (Auriv.). 46 (Kukis-wum-chorr): 3 exx 10.7.1987, 48 (700 m): 3 exx 15.7.1991.

Entephria nobiliaria (H.-S.). 12: 1 ex 1930 (Kontuniemi leg.).

Entephria caesiata (Den. et Schiff.). 2, 3, 4, 5, 6, 8, 9, 10, 12, $13,15,17,18,19,23,27,28,30,31,38-10,38-16,42 \mathrm{a}$, $43-29,43-23,43-17,43-16,43-14,43-3,44,45,46$, $48,49,50,55,56,56 \mathrm{a}, 60,61,62,71,73,78$.

Lampropteryx suffumata (Den. et Schiff.). 8, 9, 10, 12, 18, 21, 28, 37, 48, 58a, 65, 73.

Cosmorhoe ocellata (L.). 58a: 2 exx 3-4.7.1993. Northernmost record in Fennoscandia. The specimens are melanic; the white colour of forewings is dull whitish grey. One similar specimen from Karelia (Tjudi, east of Lake Onega) is kept in MZH.

Eulithis prunata (L.). 55: 1 ex 25.7.1991.

Eulithis testata (L.). 43-41: 2 exx 5.8.1993, 44: 1 ex 30.7.1980, 62: 4 exx 11.7.1987.

Eulithis populata (L.). 5, 6, 8, 9, 10, 12, 18, 19, 23, 28, 31, $33,34,37,38-16,43-29,43-17,43-7,44$ (up to subalpine birch forest), $46,48,49,50,56,56 a, 60,62$, 77,78 .

Ecliptopera silaceata (Den. et Schiff.). 10 (Sotavalta, unpubl.), 30, 58a. 
Chloroclysta infuscata (Tengstr.). 12, 18, 28, 38-16, 43 $29,43-23,43-16,45,53,55,60,64 a, 76$.

Chloroclysta truncata (Hufn.). 10, 12, 43-29, 43-18, 44 , $45,55,56,60,61$.

Chloroclista citrata (L.). 43-29: 2 exx 4-8.8.1992 (baittrap), 48: 1 ex 14.8.1984, 60: 1 ex 19.7.1986.

Thera serraria (Lienig et Z.). 27 (Krogerus 1943), 28, 30 , $37,43-29,45,48,56 a, 60,71$.

Thera variata (Den. et Schiff.). 43-20, 44, 48, 61.

Colostygia turbata (Hb.). 8, 9, 10, 12, 17, 19.

Hydriomena furcata (Thnbg.). 77 (ex 1. on Salix): 4 exx 25.7.1985.

Hydriomena impluviata (Den. et Schiff.). 58a: 1 ex 4.7.1993, 39: 2 exx 22.6.1993, 68: 1 ex 21.6.1985.

Hydriomena ruberata (Freyer), 8, 9, 10, 12, 15, 18, 23, 27, $28,29,30,36,37,39,43-29,43-3,44,48,55,56 a$, $63,65,67,73,76$.

Spargania luctuata (Den, et Schiff.). 10, 28, 39, 43-29, 43-20, 44, 46, 48, 52, 53, 55, 56, 56a, 58a, 64, 64a, 70.

Rheumaptera hastata (L.). 9, 10, 12, 15, 17, 28, 38-15, $43-29,43-20,43-7,44,45,51,55,56 \mathrm{a}, 60,63,69$, 72,73 .

Rheumaptera subhastata (Nolcken). 1, 2, 3, 5, 6, 8, 9, 10, $12,13,14,15,18,21,23,28,30,31,37,38-7,38-10$, $38-13,42 \mathrm{a}, 43-29,43-23,43-20,43-14,43-7,43-5$, $44,44 a, 46,48,53,55,56,56 a, 58 a, 59,60,63,64 a$, $65,66,69,73$.

Rheumaptera undulata (L.). 48 (900 m), 75, 77.

Euphyia unangulata (Hw.). 55: 1 ex 13.6.1992.

Epirrita autunnata (Bkh.). 10, 12, 31, 37, 38-15, 38-10, $38-5,39,43-41,43-29,43-15,43-5,44,48,53,56 a$, $58 \mathrm{a}, 59 \mathrm{a}, 64 \mathrm{a}, 65$.

Psychophora sabini frigidaria (Guenée). 42a: 3 exx 12.7.1993, 46: 3 exx [10-13.7.1870] (Sahlberg leg.) \& (Kukis-wum-chorr, 900 m) 1 ex 14.7.1986 \& (Tachtarwum-chor) 1 ex 15.8.1982, 48 (900 m): 1 ex 14.7.1991, 60: 1 ex [1870] (Sahlberg leg.).

Perizoma affinitatum (Stph.). 1, 4, 5, 6, 12, 13, 15, 17, 19.

Perizoma alchemillatum (L.). 9 (Valle 1933), 46, 56, 58a.

Perizoma blandiatum (Den. et Schiff.). 1, 2, 3, 4, 6, 8, 9 , $10,12,13,15,19,23,30,31,55,58 \mathrm{a}, 60,67,70,75$, 77.

Perizoma albulatum (Den. et Schiff.). 10, 12, 23, 55, 58a, $60,61,67,69,70,75,76,77$.

Eupithecia plumbeolata (Hw.). 55: 1 ex 12.7.1991, 65: 1 ex [24.7.1899] (Montell leg.).

Eupithecia analoga Diak. 9, 18, 28 (Krogerus 1943), 58a, 64, 66.

Eupithecia abietaria (Goeze). 27 (Krogerus 1943).

Eupithecia pygmaeata (Hb.). 9, 12, 46, 55, 56, 60, 61, 64, $64 \mathrm{a}, 66$.

Eupithecia intricata (Zett.). 3, 4, 8, 9, 10, 15, 18, 21, 36, $43-29,44,46,48,52,55,59 \mathrm{a}, 65,68,77$.

Eupithecia veratraria (H.-S.). 65: 1 ex [1899] (Montell leg., in $\mathrm{MZH}$ ). This is the only record of the species from Northern Fennoscandia. The nearest finds are from the Baltic (Latvia 19th century); generally distributed in mountain systems from West-Europe to the Pacific (Mikkola et al. 1989).
Eupithecia satyrata (Hb.). 1, 2, 3, 6, 8, 9, 10, 12, 15, 18, $21,23,28,30,31,36,38-15,38-11,39,40,43-29$, $43-20,43-15,43-7,44,44 a, 46,48,55,56,56 a, 58 a$, $61,63,64 \mathrm{a}, 65,67,73,77$.

Eupithecia tripunctaria (H.-S.). 56: 1 ex 12.6.1992. The northernmost record in Eastern Fennoscandia. This species is generally distributed in Southern Finland and Karelia up to the province Kon (Mikkola et al. 1989).

Eupithecia vulgata (Hw.). 8, 58a, 59a, 64a, 70, 75.

Eupithecia gelidata Möschl. 2, 4, 8, 9, 10, 12, 15, 17, 18, $21,27,28,30,31,38-15,38-11,39,42 a, 43-29,43-$ $20,43-15,44,44 a, 46,48,52,53,60,64 a, 65$.

Eupithecia indigata (Hb.). 18 (Valle 1933).

Eupithecia virgaureata Doubl. 3, 5, 6, 8, 9, 10, 12, 13, 15, $18,21,28,30,32,37,38-15,38-11,43-29,44,55$, $56,58 \mathrm{a}$.

Eupithecia pusillata (Den. et Schiff.). 8: 1 ex 5.7.1931.

Eupithecia lariciata (Freyer). 30: 1 ex 7.7.1964 (K. Mikkola leg.). Monophagous on Larix, which does not grow natively in this area (Hultén 1971). The only record from Northern Fennoscandia.

Eupithecia conterminata (Lienig et Z.). 12, 28 (Krogerus 1943), 43-20, 43-7, 67, 73.

Carsia sororiata (Hb.). 5, 8, 9, 10, 12, 17, 18, 19, 27, 28, $31,34,38-16,43-20,43-15,44$ (up to $600 \mathrm{~m}$ ), 46, 49, 50,60 .

Acasis appensata (Ev.). 8, 10, 21, 58a, 73.

Lomaspilis marginata (L.). 28 (Krogerus 1943), 43-20, $55,56,58 \mathrm{a}, 61,63,64 \mathrm{a}, 67$.

Semiothisa notata (L.). 10 (Sotavalta, unpubl.), 27 (Krogerus 1943), 28: 1 ex [1939] (Platonoff leg.).

Semiothisa liturata (Cl.). 10 (Sotavalta, unpubl.), 28 (Krogerus 1943).

Semiothisa clathrata (L.). 58a, 67 (Valle 1933).

Semiothisa carbonaria (Cl.). 9, 10, 12, 19, 23, 28, 29, 31, $37,38-14,39,43-41,43-25,43-9,43-5,44$ (up to $500 \mathrm{~m}), 60,63,68,73,77$.

Itame loricaria (Ev.). 55 (ex 1. from Betula): 1 ex 20.7.1991. Itame wauaria (L.). 55: 1 ex 25.7.1991.

Itame brunneata (Thnbg.). 12, 18, 28, 37, 38-16, 43-29, $43-20,43-15,44$ (up to $500 \mathrm{~m}$ ), 46, 55, 56, 60, 61, 62, $64 \mathrm{a}$.

Pygmaena fusca (Thnbg.). 1, 2, 4, 6, 8, 10, 12, 14, 15, 19, $23,28,31,36,43-23,44,46$ (up to Kukis-wum-chorr, $900 \mathrm{~m}), 60,65,77$.

Plagodis pulveraria (L.). 64a: 1 ex 4.7.1993. Northernmost record from Fennoscandia.

Hypoxystis pluviaria (F.). 67 (Valle 1933).

Selenia dentaria (F.). 4, 6, 8, 9, 10, 12, 18, 21, 23, 27, 28, 31, $36,37,43-29,44,46,48,52,56,56 a, 60,67,68,73$.

Selenia tetralunaria (Hufn.). 58a: 1 ex 4.7.1993, 68: 1 ex 21.6.1985.

Epirrantis diversata (Den. et Schiff.). 9: 3 exx 21.5.1934, 17: 8 exx (Carpelan leg.), 37: 1 ex [1899] (Poppius leg.).

Lycia pomonaria (Hb.). 9, 10, 12, 17, 29 (Valle 1933), 37.

Lycia lapponaria (Boisd.). 10: 1 ex 11.5.1979, 44a (450 m): 1 ex 22.5.1975, 46 (Valle 1933). 
Ematurga atomaria (L.). 4, 6, 8, 10, 12, 15, 23, 28, 38-7, $38-10,38-13,39,42 \mathrm{a}, 43-29,43-23,43-20,43-17$, $43-14,43-11,43-9,43-1,44,44 a, 45,48,55,56$, $58 \mathrm{a}, 59 \mathrm{a}, 60,63,64 \mathrm{a}, 67,68,73,77$.

Cabera pusaria (L.). 64a: 5 exx 4-21.7.1993.

Cabera exanthemata (Scop.). 59a, 61, 63, 64a, 67, 70.

Hylaea fasciaria (L.). 61: 1 ex 28.7.1991.

Parietaria vittaria (Thnbg.). 1, 2, 3, 4, 5, 6, 8, 9, 10, 12, 14, $15,18,21,23,28,31,34,38-8,38-16,39,43-29,44$, 44a, 48, 52, 55, 56a, 58a, 60, 64, 65, 67, 73.

Glacies coracina (Esp.). 1, 2, 6, 8, 10, 12, 14, 15, 23, 36, $42 \mathrm{a}, 44(400-700 \mathrm{~m}), 44 \mathrm{a}, 46$ (up to Kukis-wumchorr, $900 \mathrm{~m}), 48,60,65$.

\section{Lasiocampidae}

Poecilocampa populi (L.). 56: 1 ex 20-27.7.1992 (light trap).

Trichiura crataegi (L.). 10: 1 ex 23.7.1980, 12 (Valle 1933), 46 (Fridolin 1935).

Eriogaster lanestris (L.). 18 (Valle 1933), 20 (Nordström et al. 1961).

Cosmotriche lunigera (Esp.). 28 (Krogerus 1943).

Phyllodesma ilicifolium (L.). 60 (Nordström et al. 1961).

\section{Saturniidae}

Saturnia pavonia (L.). 8, 10, 12, 28 (Krogerus 1943), 43$19,43-9,44,46,77$.

\section{Sphingidae}

Acherontia atropos (L.). 3 (Nordström et al. 1961), 56 (Novikov 1958).

Hyles gallii (Rott.). 10 (Nordström et al. 1961), 27 (Krogerus 1943), 39: 1 ex 22.7.1992.

\section{Notodontidae}

Furcula furcula (CI.). 12, 21, 28.

Notodonta dromedarius (L.). 6 (Sotavalta, unpubl.), 9 (Vaile 1933), 46 (Fridolin 1935).

Eligmodonta ziczac (L.). 27 (Krogerus 1943), 28, 46 (Fridolin 1936), 60, 68.

Pheosia gnoma (F.). 3, 8, 10 (Valle 1933), 12, 43-9, 56.

Ptilodon capucina (L.). Lapponia Rossica (Tengström, unpubl.; no later confirmation).

Clostera pigra (Hufn.). 2, 28 (Krogerus 1943), 46 (Fridolin 1936), 55.

\section{Lymantriidae}

Calliteara fascelina (L.). 46 (Kozhanchikov 1950).

Leucoma salicis (L.). 23, 46, 48, 56.

\section{Arctiidae}

Setina irrorella (L.). 2, 12 (Valle 1933), 45 (Nordström et al. 1961).
Holoarctia fridolini (Torst.) 46: 2 exx 22-23.7.1926, 1 ex August 1931. Mentioned by N.Kusnezov (1935) under the name Chibinarctia fridolini, which is nomen nudum according to the International Code of Zoological Nomenclature. The problems connected with this species were described by Torstenius (1971) who gave a formal description of fridolini, but considered it as a subspecies of O. cervini Fallou. Ferguson (1984) considers $H$. fridolini as an independent species.

Parasemia plantaginis (L.). 8, 10, 27, 28, 43-20, 43-16, $56,60,67,71,73,77$.

Pararctia lapponica (Thnbg.). 3, 6, 8, 9, 10, 12, 21, 28.

Diacrisia sannio (L.). 70: 1 ex 15.7.1992, 76: 1 ex 15.7.1992.

Phragmatobia fuliginosa (L.). 4, 8, 9, 10, 12, 15, 18, 28, $36,44$.

\section{Noctuidae}

Polypogon tentacularius (L.). 48 (Raijavr), 65, 67, 69, 70, $71,75$.

Hypenodes humidalis Doubl. 62: 1 ex 11.8.1987.

Scoliopteryx libatrix (L.). 46 (Fridolin 1936), 78, 79 (Nordtsröm et al. 1969).

Euclidia glyphica (L.). 12 (Nordström et al. 1969), 44, 60, $64 \mathrm{a}, 67$.

Nola karelica Tengstr. 9 (Valle 1933), 27 (Krogerus 1943), 28 (Nordström et al. 1961).

Plusia festucae (L.). 39: 1 ex 27.7.1991 (bait-trap).

Autographa macrogamna (Ev.). 12 (Sotavalta, unpubl.), 55: 1 ex 25.7.1991, 56: 1 ex 18.7-5.8.1992 (lighttrap), 61: 1 ex 28.7.1991.

Autographa gamma (L.). 9 (Valle 1933).

Syngrapha diasema (Boisd.). 9, 10 (Valle 1933), 14, 17, 28 (Krogerus 1943), 43-7, 50, 56, 60.

Syngrapha microgamma (Hb.). 27 (Krogerus 1943), 4320: 1 ex 15.7.1991.

Syngrapha interrogationis (L.). 12, 14, 28, 43-7, 46, 47, 48, 49, 50, 56 (light-trap), 57, 65, 66, 74, 77, 78, 79.

Syngrapha parilis (Hb.). 6, 9, 10, 12, 14, 17, 28, 31, 36, 58.

Caloplusia hochenwarthi (Hochenw.). 1, 2, 6, 9, 10, 12, $15,19,23,28,31,73$.

Acronicta psi (L.). 76: 1 ex 15.7.1992 (bait-trap).

Acronicta menyanthidis (Esp.). 63: 1 ex 27.6.1981, 76: 2 exx 15.7.1992 (bait-trap).

Acronicta auricoma (Den. et Schiff.). 4, 9, 10, 12, 28, 36, $38-16,43-29,43-23,43-21,43-20,43-16,43-7$, $43-3,44,56 a, 60,61$.

Enargia paleacea (Esp.). 43-35: 1 ex 7.8.1993, 43-29: 1 ex 7.8.1993, 43-16: 2 exx 4-8.8.1992, 43-5: 1 ex 12.8.1993 (all from bait-traps).

Parastichtis suspecta (Hb.). 10: 2 exx 9-19.7.1979, 56a: 1 ex 7.8.1993 (bait-trap).

Hyppa rectilinea (Esp.). 12, 21, 28, 39, 43-29, 43-23, 4321, 43-16, 43-14, 43-1, 44, 76.

Apamea crenata (Hufn.). 43-7: 1 ex 18.7.1992 (bait-trap). Apamea lateritia (Hufn.). 43-18: 1 ex 29.7.1992 (baittrap), 55: 1 ex 25.7.1991. 
Apamea maillardi (Geyer). 39: 1 ex 28.7.1993, 43-29: 1 ex 31.7.1992 \& 1 ex 12.8.1993, 43-23: 1 ex 31.7.1992, 43-18: 5 exx 27-31.7.1992, 43-16: 1 ex 1-4.8.1992 \& 3 exx 28.7-7.8.1993, 43-7: 1 ex 4-8.8.1992 (all from bait traps).

Apamea remissa (Hb.). 12, 34, 39, 43-5.

Caradrina clavipalpis (Scop.). 12, 60, 63 (Nordström et al. 1969), 66.

Hillia iris (Zett.), 6 (Valle 1933), 15 (Nordström et al. 1969), 43-29: 1 ex 2-7.8.1992 (bait-trap), 77 (ex 1. from Salix): 1 ex 25.7.1985.

Sympistis funebris (Hb.). 10, 12, 14, 18, 23 (Miller 1915), 28 (Krogerus 1943), 36, 38-16, 44, 48 (600 m), 52, $58,60,64 a$.

Sympistis heliophila (Payk.). 1, 2, 3, 4, 5, 6, 8, 9, 10, 12, $15,18,19,23,28,29,31,36,38-7,38-10,38-13,38-$ $16,42 a, 43-29,43-23,43-21,43-16,43-14,43-9$, $43-5,44,44 a, 46$ (up to Kukis-wum-tschorr, $900 \mathrm{~m}$ ), $48,52,56,56 \mathrm{a}, 58 \mathrm{a}, 60,6 \mathrm{I}, 67$.

Sympistis lapponica (Thnbg.). 6, 8, 10, 12, 14, 15, 34, 44a, 46 (up to Kukis-wum-chorr, $900 \mathrm{~m}$ ), 48.

Sympistis zetterstedtii (Stgr.). 8, 12, 42a, 46 (up to Kukiswum-chorr, $900 \mathrm{~m}), 48$.

Dasypotia templi (Thnbg.), 6: 1 ex June 1929 (Pohjakallio leg.).

Lithomoia solidaginis (Hb.). 56: 1 ex 11.8.1985, 62 (sec. Peltonen 1947), 66 (Sotavalta, unpubl.).

Litophane lambda (F.). 28: 1 ex [1939] (Platonoff ]eg.).

Xylena vetusta (Hb.). 12: 1 ex 17.6.1933, 46 (Novikov 1958).

Mniotype adusta (Esp.). 43-23: 1 ex 18.7.1991, 43-17: 2 exx 28.7-2.8.1993, 43-14: 1 ex 8.7.1991, 64a: 1 ex 21.7.1993 (bait-traps).

Anarta myrtill (L.). 12, 28, 38-16, 38-11, 43-5, 44, 46 (Kukis-wum-chorr, $900 \mathrm{~m}$ ) (Fridolin 1935), 48, 52, $56,58 \mathrm{a}$

Anarta cordigera (Thnbg.). 3, 5, 6, 8, 9, 10, 12, 18, 21, 23 , $28,34,36,38-15,43-20,44$ (up to $600 \mathrm{~m}$ ), 46 (up to Kukis-wum-chorr, $900 \mathrm{~m}), 52,60$.

Anarta melanopa (Thnbg.). 1, 4, 5, 6, 8, 9, 10, 12, 14, 15, $23,31,36,39,42 a, 44,44 a, 46$ (up to Kukis-wumchorr, $900 \mathrm{~m}), 48,52,60$.

Lasionycta staudingeri (Auriv.). 12 (Valle 1933), 14, 42a, 60,65 .

Lasionycta skraelingia (H.-S.). 27 (Krogerus 1943).

Hada nana (Hufn.). 10 (Sotavalta, unpubl.), 15 (Nordtsröm et al. 1969), 28 (Sotavalta, unpubl.).

Anartomima secedens bohemani (Stgr.). 8, 10 (Valle 1933), 14,45 .

Polia richardsoni (Curt.). 12 (Valle 1933), 14, 60, 65.

Polia lamuta (Hertz), 60: 1 ex 7.7.1913.

Polia conspicua sabmeana Mikkola. 38-1: 9 exx 13.72.8.1993, 42a: 1 ex 18.7.1993, 43-1: 13 exx 1227.7.1991, 43-5: 1 ex 23.7.1991 \& 18 exx 1828.7.1993 (bait-traps), 46 ("Chibiny [railway] station"): 1 ex 23.7.1926 (deposited in Zoological Museum of Moscow University, Russia; K.Mikkola det.). This newly described (Mikkola 1980) subspecies of a Siberian noctuid is fairly common on the low felis of Inari Lapland on the Finnish side of the border.
Melanchra pisi (L.). 43-16: 1 ex 18.7.1992 (bait-trap), 55 (Znamenskaja 1962).

Papestra biren (Goeze). 8, 10, 12, 14, 15, 21, 27, 31, 36, 38-11, 43-3, 43-29, 44 (600 m), 46 (Kukis-wumchorr) (Fridolin 1935), 60.

Cerapteryx graminis (L.). 14, 15 (Valle 1933), 43-35, 55, $63,66$.

Diarsia mendica (F.). 1, 2, 3, 6, 8, 9, 10, 12, 14, 15, 18, 28, $39,43-29,43-23,43-21,43-16,43-14,43-9,43-5$, $43-1,44,46$ (up to Kukis-wum-chorr, $900 \mathrm{~m}$ ), 56, $56 a, 61,62,71,73$.

Xestia quieta (Hb.). 1, 2, 6, 8, 11 (Nordström et al. 1969), 12, 15, 23 (Miller 1915).

Xestia rhaetica (Stgr.). 43-29: 8 exx 19-27.7.1991, 29 exx 23.7-8.8.1992, 3 exx 28.7.1993, 43-23: 1 ex 31.7.1992, 43-20: 1 ex 31.7.1992, 56a: 14 exx 23.7-7.8.1993 (bait-traps), 64a.

Xestia speciosa (Hb.). 1, 10, 12, 14, 15, 28, 37, 43-16, 433, 46 (Kukis-wum-chorr) (Fridolin 1935), 56a, 71, 73.

Xestia borealis (Nordström). 45: 1 ex [8-9.7.1870] (Sahlberg leg., in MZH). This specimen was described as Agrotis imandrensis (Lingonblad 1933).

Xestia distensa (Ev.). 28 (abundant in 1939; Krogerus \& Platonoff leg.), 56a: 1 ex 28.7.1993 (bait-trap), 73: 1 ex [1887] (Edgren leg.). This and the following species have been considered to be conspecific, so the old literature records, which usually concern $X$. laetabilis Zett., are not reliable. The specific difference was shown by Suomalainen (1983).

Xestia laetabilis (Zett.). 48: 1 ex [1887] (Palmén leg.), 56a: 1 ex 18.7.1993 (bait-trap), 71: 1 ex [1887] (Edgren \& Levander leg.), 73: 1 ex [1887] (Edgren leg.). K.Mikkola (pers.comm.) investigated also 5 males in coll. Tsvetajev (kept in Zoological museum, Moscow): "Lapp. ross. spt. v. Terr. Murmanensis, stat. Shongui, S. Tshetverikov leg. 21-23.7.1928".

Xestia alpicola (Zett.). 2, 4, 8, 9, 10, 15 (Valle 1933), 17 , $18,19,28,38-16,38-11,43-41,43-29,43-20,43-$ $16,43-9,44$ (up to $600 \mathrm{~m}$ ), 56a, 64a, 76.

Xestia lorezi kongsvoldensis (Grönl.). 2, 8, 12, 13, 15.

Xestia tecta (Hb.). 1, 5, 8, 10, 12, 14, 15, 27 (Krogerus 1943), 37, 43-29, 46.

Eurois occultus (L.). 25, 26 (Nordström et al. 1969), 38$16,43-29,43-18,43-5,46,48,56,56 a, 71,73$.

\section{Discussion}

\subsection{Level of our knowledge}

Compared to other areas in northern Fennoscandia, the lepidopteran fauna of the Kola Peninsula is still rather poorly known, especially the so called "Microlepidoptera".

The number of species in localities (Table 1) and UTM squares (Fig. 4) clearly show that only 


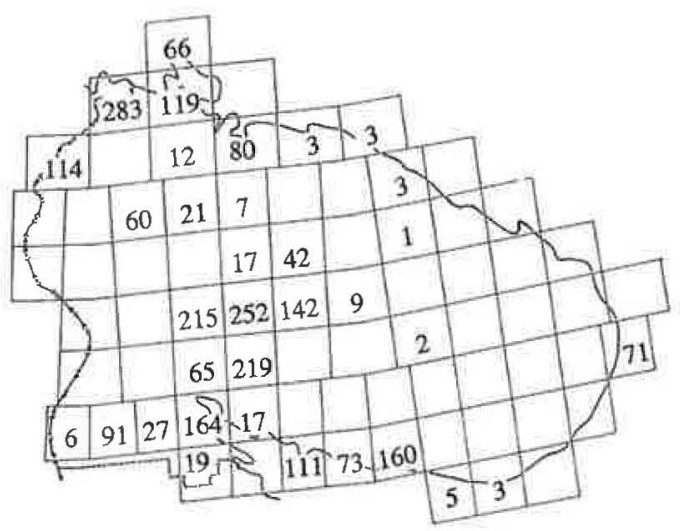

Fig. 4. Number of species summarized for UTM squares.

Petsamo area and Chibiny mountains (with about 250-300 species in each) are more or less satisfactory investigated. Comparing with the communes of Utsjoki $\left(5200 \mathrm{~km}^{2}, 439\right.$ species recorded) and of Inari $\left(17500 \mathrm{~km}^{2}, 444\right.$ species recorded) (Koponen et al. 1982), we suppose that local faunas in this region should be at the level of 250-400 species.

Linnaluoto \& Koponen (1980) listed 602 species from northern Fennoscandia (including northern Norway, province Li of Finland, northern Petchenga and the Kilpisjärvi area in northwestern corner of Finland). This list includes about 150 species not recorded from the Kola Peninsula, major part of which are still with certainty to be found. For example, the species flying in early spring and late autumn are poorly known. We would also like to emphasize that the fauna of the northeastern tundra, all the way from Murmansk to Ponoj, is entirely unknown and that research in this area would certainly offer zoogeographically important finds.

Among the 585 species included in the list, records of 32 species are based on data from previous publications only. For all other species we have seen some materials.

\subsection{New records and deletions}

Seven species are not found elsewhere in Northern Fennoscandia. Among them, 5 species are widely distributed southwards from this region [Bucculatrix maritima Stt., Elachista monosemiella Rössl., Scythris obscurella (Scop.), Eupoecilia sanguisorbana (H.-S.), Pammene aurana (F.)] and 2 other [Dichrorampha cinerascens (Danil.), Clepsis rogana (Gn.)] are eastern species. Six of them are occuring on the White Sea coast, and we expect more interesting records from this poorly investigated area.

The record of Hypsopygia costalis (F.) is most probably based on a migrated specimen.

Since no complete lists were published before, it is quite difficult to calculate the exact number of species which have never been reported from this region. Moreover, we believe that it is not necessary. Anyway, 585 species are recorded from the whole area; 285 of them were reported from Petsamo (Sinev 1988). Taking into account other publications, we suppose that about 100 species in our list are new for the Kola Peninsula.

Although there are no check-lists of the Russian fauna, comparison with recently published keys of European moths (e.g. Kuznetzov 1978, Piskunov 1981) enabled us to state that at least eight species have never been reported from the Russian territory. One of them, Bucculatrix maritima Stt. is widely distributed in the temperate regions of Europe. The other seven species are Northern Fennoscandian ones, described in the last decades (Coleophora unigenella Svensson, C. boreella Bengtsson, Scythris fuscopterella Benander, Aproaerema karvoneni Hackman, Acompsia subpunctella Svensson, Sophronia gelidella Nordman, Catastia kistrandella Opheim).

The following species are excluded from the list:

Nematopogon metaxellus (Hb.). Specimen mentioned by Valle (1933) belongs to N. pilellus (Den, et Schiff.).

Elachista poae Stt. 12 (Valle 1933). We did not find specimens mentioned by K.J.Valle. Old meaning of the name "poae" refer to the complex of northern large black-white Elachista species.

Cnephasia virgaureana Tr. 44 (Kozlov 1987). Mislabelled specimens.

Acleris notana (Don.). 18 (Valle 1933). The specimens are kept in collection of E.Suoma- 
lainen; they proved to be A. implexana ferrumixtana (Benander).

Agonopterix arctica (Strand). 12 (Linnaluoto \& Koponen 1980). Record is based on the doubtful determination of Valle (1933); no specimens has been found.

Thera obeliscata (Hb.). Kola region (Mikkola et al. 1989). Erroneous record based on a specimen collected in Finland, NE part of the province Lkem.

Thera juniperata (L.). Same as T. obeliscata.

Plebejus argus (L.) We have not seen any specimens from the Peninsula. In the old lists only Fridolin (1936) mentioned this species. On the other hand, he did not include Lycaeides idas (L.) which is not rare in this area. Thus, the occurence of $P$. argus (which is generally distributed southwards of this area) on the Kola Peninsula is uncertain.

Acknowledgements. M. Kozlov thanks all the persons who joined him during expeditions to Kola and helped him to collect material in the harsh environmental conditions: Alexandr Kravtshuk, A. Lvovsky, Irina Sokolova, L. Svetsova, Elena Zvereva and Vitaly Zverev. Expeditions to Kola in 1991-1993, as well as the completing of this paper, were funded by the Academy of Finland (project leaders Dr. Kauri Mikkola and Prof. Erkki Haukioja). A. Lvovsky and S. Sinev kindly assisted us with search of the specimens in the collections of ZIN and provided the necessary information. Some delails of collecting trips of K. J. Valle to Petsamo area, as well as taxonomical problems connected with Arctiidae, were clarified by E. Suomalainen. We are greatly indebted to $\mathrm{H}$. Silfverberg for consultations on old Finnish collections, and to colleagues who helped us in the determination of some problematic groups, namely V. I. Kuznetzov (ZIN), L. Kaila and J. Kullberg (MZH). Kauri Mikkola provided us with valuable information on Noctuidae and Geometridae and made numerous helpful comments to the drafts of the paper. Special thanks are due to Mrs A. Albrecht, L. Huldén and V. Varis for help during preparation of the manuscript.

\section{References}

Aagard, K. 1979: Sommerfugler i Nord-Norge. — Ottar 113/114:1-65.

Bengtsson, B. $\AA$. 1977: Two new species of Microlepidoptera from northern Sweden (Lepidoptera: Elachistidae, Scythrididae). - Entomol. Scand. 8:55-58.

Dang, P. T. 1992: Holarctic distribution of Choristoneura albaniana (Walker), with new synonymy (Tortricidae). - J. Lepidopterol. Soc. 46:269-272.
Djakonov, A. М. (Дьяконов, А. М.) 1911: [Notes on the Lepidoptera of Murmansk coast] (In Russian) - Russian Entomol. Rev. 11(1):13-17.

Ferguson, D. C. 1984: Contributions towards reclassification of the world genera of the tribe Arctiini. Part 1 Introduction and revision of the Neoarctia - Grammia group (Lepidoptera: Arctiidac; Arctiinae). - Entomography 3:181-275.

Fridolin, V. Ju. (Фридолин, В.Ю.) 1935: Koukiss-woumtschorr, le plateau central des Monts Khibines au point de vue biocenotique. - Entomol. Obozrenie 26(1/ 4):165-182.

- 1936: [Community of animals and plants of Chibiny mountain area. Biocenothical investigations in 19301935]. (In Russian) - Izd. AN SSSR, Moscow \& Leningrad. 295 p.

Gribova, S. A., Isachenko, T. I. \& Lavrenko, E. M. (Грибова, С. А., Исаченко, Т. И. \& Лавренко, Е. M.) 1980: [Vegetation of the European part of USSR]. (In Russian) - Nauka, Leningrad. 420 p.

Gustafsson, B. (ed.) 1987: Catalogus Lepidopterorum Sueciae. - Naturhistoriska Riksmuseet, Stockholm.

Hultén, E. 1971: Atlas över växternas utbredning i Norden. - Generalstabens Litografiska Anstalts Förlag, Stockholm. 531 p.

Itämies, J. \& Kyrki, J. 1983: Biology and larva of Aproaerema karvoneni (Lepidoptera, Gelechiidae).-Notulae Entomol. 63:127-130.

Jalas, J. \& Suominen, S. (eds.) 1972: Atlas Florae Europaea. 1. Pteridophyta (Psilotaceae to Azollaceae). - The Committee for Mapping the Flora of Europe \& Soc. Biol. Fennica Vanamo, Helsinki. 121 p.

Jalava, J. 1977: The female of Coleophora unigenella (Lepidoptera, Coleophoridae). - Notulae Entomol. 57:70.

Jalava, J. \& Kyrki, J. 1980: Notes on the taxonomy and distribution of western Palaearctic Trichophaga species (Lepidoptera, Tineidae). - Notulae Entomol. 60:107-110.

Johansson, R. 1967: Coleophora thulea n. sp. - Opuscula Entomol. 32:135-138.

Kaila, L. 1992: The Elachistidae of southern Siberia and Central Asia, with descriptions of five new species (Lepidoptera). - Entomol. Fennica 3:177-194.

Kaila, L. \& Kerppola, S. 1992: Elachista leifi sp.n. from northern Finland, - Entomol. Fennica 3:155-158.

Koponen, S., Laasonen, E.M. \& Linnaluoto, E.T. 1982: Lepidoptera of Inari Lapland, Finland. - Kevo notes 6:1-36.

Kotzsch, H. 1933: Auf Schmetterlingsjagd am nördlichen Eismeer. - Entomol. Zeitung. 47:142-145, 152-155.

Kozhanchikov, I. V. (Кожанчиков, И. В.) 1950: [Orgyidae. Fauna of the USSR. Lepidoptera. Vol. 12]. (In Russian). - Izd. AN SSSR, Moscow \& Leningrad. $581 \mathrm{p}$.

Kozlov, M. V. (Kозлов, M. B.) 1981a: [Butterflies (Lepidoptera, Rhopalocera) of Kola peninsula and Karelia]. (In Russian) -- In: [Biological problems of the North. IX Symposium: Abstracts]: 184. Syktyvkar. 339 p. 
- 1981b: [Zeiraphera griseana on spruce]. (In Russian) - Zashchita Rastenii [Plant Protection] (Moscow) (12):45.

- 1983a: [Butterflies (Lepidoptera, Rhopalocera) of Murmansk region]. (In Russian) - VINITI no. 3188 83Dep. 16 p.

- 1983b: The primitive moths (Lepidoptera: Micropterigidae, Eriocraniidae) of North-West regions of U.S.S.R. (In Russian with English summary) - Biologicheskie Nauki [Biol. Sci.] (Moscow) (11):32-36.

- 1983c: [Lepidoptera of mountain tundra of Kola peninsula]. (In Russian) - In: [Biological problems of North. X Symposium: Abstracts, pt. 2]: 356-357. Magadan. 462 p.

- 1984a: [Birch-feeding Lepidoptera in Murmansk region]. (In Russian) - Zashchita Rastenii [Plant Protection] (Moscow) (4):31-32.

- 1984b: The role of insects in plant communities of Kola peninsula. (In Russian) - In: [Protection of plant communities in northern regions. Proc. of AllUnion Conference, Pt 1]: 86-89. Syktyvkar. 175 p.

- 1987: [Leafrollers (Lepidoptera, Tortricidae) as plant pests in Murmansk region]. (In Russian) - In: Sinadsky, Yu. V. (ed.), [Migration of pest organisms caused by plant introduction]: 33-38. Apatity. 98 p.

Krogerus, H. 1943: Lepidopterologiska studier i södra Petsamo. - Notulae Entomol. 23:19-43.

- 1972: The invertebrate fauna of the Kilpisjärvi area, Finnish Lapland. 14. Lepidoptera. - Acta Soc. Fauna et Flora Fennica 80:189-222.

Kryuchkov, V. V. (Крючков, В. В.) 1991: [Limits of anthropogenic loads and the state of ecosystems of the North]. (In Russian) — Ecologia (Sverdlovsk) (3):2840.

- 1993: Degradation of ecosystems around the "Severonikel" smelter complex, - In: Kozlov, M. V., Haukioja, E. \& Yarmishko, V. T. (eds.), Aerial pollution in the Kola peninsula. Proc. Intern. Workshop, April 14-16, 1992, St. Petersburg: 35-46. Apatity. 417 p.

Kuznezov, N. J. 1935: The origin of the Lepidopterous fauna of the Arctic Eurasia (preliminary note). Arctica 3:115-136.

Kuznetsov, V. I. (Кузнецов, В. И.) 1978: [Fam. Tortricidae (Olethreutidae, Cochylidae) - Leafrollers]. (In Russian) - In: Medvedev, G. S. (Медведев, Г. С.) (ed.), [Key for determination of insects of the European part of USSR. Vol. 4. Lepidoptera, pt. 1.]: 193-680. Nauka, Leningrad. $711 \mathrm{p}$.

Kuznetsov, V. I. \& Jalava, J. 1988: Soviet-Finnish entomological expeditions to Southern Siberia 1982-1984. 2. Tortricidae (Lepidoptera). - Nota lepid. 11(2):126138.

Kyrki, J. 1978: [Distribution of Finnish microlepidoptera. I. Fauna of biological provinces (Lepidoptera: Micropterigidae - Pterophoridae]. (In Finnish) - Notulae Entomol. 58:37-67.

- 1979: [Distribution of Finnish microlepidoptera. II. Additions to the fauna of biological provinces (Lepi- doptera: Micropterigidae - Pterophoridae)]. (In Finnish) - Notulae Entomol. 59:125-131.

- 1981a: [Nemophora amurensis in East Fennoscandia (Lepidoptera, Incurvariidae)]. (In Finnish) - Notulae Entomol. 61:125-129.

- 1981b: The larval case and food plant of Coleophora thulea (Lepidoptera, Coleophoridae). - Notulae Entomol. 61:161-163.

- 1988: Reassesment of the genus Rhigognostis Zeller, with descriptions of two new and notes on further seven Palaearctic species (Lepidoptera, Plutellidae). — Entomol. Scand. 19:437-453.

Kyrki, J. \& Karvonen, J. 1984: The biology of Coleophora unigenella (Lepidoptera, Coleophoridae). - Notulae Entomol. 64:51-53.

Laasonen, E. 1980: [Lepidoptera of Kuhmo, Kuusamo and East Lapland 1980]. (In Finnish) - Baptria 5(4):8587.

Laasonen, E., Jalava, J. \& Schmidt Nielsen, E. 1981: The identity of Incurvaria vetulella (Zetterstedt, 1839) and I. circulelia (Zetterstedt, 1839), two distinct species (Lepidoptera: Incurvariidae). - Entomol. Scand. 12:57-64.

Lingonblad, B. 1933: Eine neue Agrotis-Art von der KolaHalbinsel. - Notulae Entomol. 13:87-89.

Linnaluoto, E. T. \& Koponen, S. 1980: Lepidoptera of Utsjoki, northernmost Finland. - Kevo notes 5:1-68.

Mikkola, K. 1980: Two new Noctuid species from Northern Europe: Polia sabmeana sp.n. and Xylomoia strix sp.n. (Lepidoptera, Noctuidae: Hadeninae and Amphipyrinae). - Notulae Entomol. 60:217-222.

Mikkola, K., Jalas, J., Peltonen, O. 1985: [Lepidoptera of Finland. Geometridae 1. Geometroidea 1]. (In Finnish) - Suomen perhostutkijain seura ry., Helsinki. $260 \mathrm{p}$.

- 1989: [Lepidoptera of Finland. Geometridae 2]. (In Finnish) - Suomen perhostutkijain seura ry., Helsinki. 280 p.

Miller, Е. (Миллер, Е,) 1915: [On the characteristic of lepidopteran fauna of Murmansk coast]. (In Russian) - Bull. Soc. Entomol. Moscow 1:124-135.

Nielsen, E. S. \& Johansson, R. 1980: Cauchas breviantennella n.sp. from NW Europe and C. brunnella n.sp. fiom Uzbekistan, with a check-list of the Palaearctic Cauchas species (Lepidoptera: Adelidae). — Entomol. Scand. 11:145-153.

Nordström, F., Opheim, M. \& Valle, K.J. 1955: De fennoskandiska dagfjärilatnas utbredning. Lepidoptera Diurna (Rhopalocera \& Hesperioidea) [Distribution of Fennoscandian butterflies]. (In Swedish) — Lunds Univ. Årsskrift 51(1):1-177.

Nordström, F., Opheim, M. \& Sotavalta, O. 1961: De fennoskandiska svärmarnas och spinnarnas utbredning (Sphinges, Bombycomorpha etc.) [Distribution of Fennoscandian moths]. - Lunds Univ. Årsskrift 57(4): 1-92, maps 1-181.

Nordström, F., Kaaber, S., Opheim, M. \& Sotavalta, O. 1969: De fenmoskandiska och danska nattflynas 
utbredning (Noctuidae) [Distribution of Fennoscandian and Danish moths]. -.. Lund, 159 pp, 403 maps.

Novikov, G. A. (Новиков, Г. А.) 1958: [Taxonomical review of the animals of the Kola Peninsula. Moths and butterflies - Lepidoptera]. (In Russian) - In: Pavlovsky, E. N. (Павловский, Е. H.) (ed.), [The world of animals of the USSR. 5. Mountain territories of the european part of the USSR]: 564-566. Izd. AN SSSR, Moscow \& Leningrad. 655 p.

Novitskaja, L. А. (Новицкая, Л.А.) 1962: [Review of plant pests in Murmansk region]. (In Russian) - In: [Decorative plants and planting of greenery in Extreme North regions of USSR]: 182-186. Nauka, Moscow \& Leningrad.

Opheim, M. 1975: The Lepidoptera of Norway. Checklist. 1. - Kongl. Norske Vidensk. Selskab Museet, Trondheim. 36 p.

- 1977: The Lepidoptera of Norway. Check-list. 2. Kongl. Norske Vidensk. Selskab Museet, Trondheim. $31 \mathrm{p}$.

- 1978: The Lepidoptera of Norway. Check-list. 3. Kong1. Norske Vidensk. Selskab Museet, Trondheim. $30 \mathrm{p}$.

Opheim, M. \& Fjeldså, A. 1980: The Lepidoptera of Norway, check list. 4. - Kongl. Norske Vidensk. Selskab Museet, Trondheim. $32 \mathrm{p}$.

Peltonen, O. 1947: Zur Schmetterlingsfauna von Viena. (In Finnish with German summary) - Ann. Entomol. Fennici 13:131-144.

Petersen, B. 1947: Die geographische Variation einiger fennoskandischer Lepidopteren. - Zool. Bidrag Uppsala. 26:329-531.

Piskunov, V. I. (Пискунов, В. И.) 1981: [Fam. Gelechiidae]. (In Russian) - In: Medvedev, G. S. (Медведев, Г. С.) (ed.), [Key for determination of insects of the European part of USSR. 4. Lepidoptera 2.]: 659-748. Nauka, Leningrad. 787 p.

Ramenskaja, M. L. (Раменская, М. Л.) 1983. [Analysis of the flora of Murmansk region and Karelia]. (In Russian) - Nauka, Leningrad. 215 p.

Razowski, J. 1970: Cochylidae. Microlepidoptera Palaearctica. 1, 1 (text). - G. Fromme \& Co. Verlag, Wien. $528 \mathrm{p}$.

Seksayeva, S. V. (Сексяева, C. В.) in press: [Revision of Bucculatricidae (Lepidoptera) of the Russia]. (In Russian) - In: Kuznetzov, V. I. (Кузнецов, В. И.) (ed.),
Proc. Zool. Inst., Russian Acad. Sci.

Silfverberg, H. 1988: Finnish entomologists on the Kola Peninsula. - Notulae Entomol. 68:115-120.

Sinev, S. Ju. (Синев, С. Ю.) 1988: On the lepidopterous fauna of the Pechenga district of the Murmansk provence. (In Russian) - In: Zlobin, V. V. (Злобин, В. B.) (ed.), The connections between entomofauna of North Europe and Siberia: 168-173. Zool. Inst., Leningrad. $188 \mathrm{p}$.

Suomalainen, E. 1983: Xestia (Anomogyna) laetabilis (Zetterstedt) and X. distensa (Eversmann) (Lepidoptera, Noctuidae): two species confused. - Notulae Entomol. 63:115-123.

Svensson, I. 1966: New and confused species of Microlepidoptera. - Opuscula Entomol. 31:183-202

af Tengström, J. M. J. 1869: Catalogus Lepidopterorum Faunae Fennicae praecursorius. - Acta Societatis Fauna Flora Fennica 10:289-368.

- 1873: [New records of Finnish Lepidoptera]. (In Swedish) - Notiser ur Sällskapets pro Fauna et Flora Fennica förhandlingar 14:29-39.

Torstenius, S. 1971: Orodemnias cervini Fallou ssp. fridolini n.ssp. (Lepidoptera, Arctiidae). - Entomol. Tidskr. 92(3/4):173-177.

Traugott-Olsen, E. \& Schmidt Nielsen, E. 1977: The Elachistidae (Lepidoptera) of Fennoscandia and Denmark. - Fauna Entomol. Scand. 6:1-299.

Valle, K. J. 1933: Die Lepidopterenfauna des Petsamogebietes unter faunistisch-zoogeographischer Berïcksichtigung der Fauna ganz N-Fennoscandias. - Ann. Zool. Soc. "Vanamo". 1(3):1-262.

Varis, V., Jalava, J. \& Kyrki, J. 1987: Check-list of Finnish Lepidoptera - Suomen perhosten luettelo. - Notulae Entomol. 67:49-118.

Vershinina, N. P. (Вершинина, Н. П.) 1981: [Pests of decorative plants in Murmansk region]. (In Russian) - In: [Development of botanical investigations in Kola area]: 138-147. Apatity.

Vuola, M. \& Korpela, S. 1977: Suomen lasisiipisten (Sesiidae) ja puuntuhoojien (Cossidae) elintavoista (Lepidoptera). 2. Raidan lasisiipi (Sesia bembeciformis) ja varjolasisiipi (Paranthrene tabaniformis). - Notulae Entomol. 57:3-8.

Znamenskaja, M. К. (Знаменская, М.К.) 1962: [Agricultural pests in Murmansk region]. (In Russian). Entomol, Obozr. 41(2):310-321. 